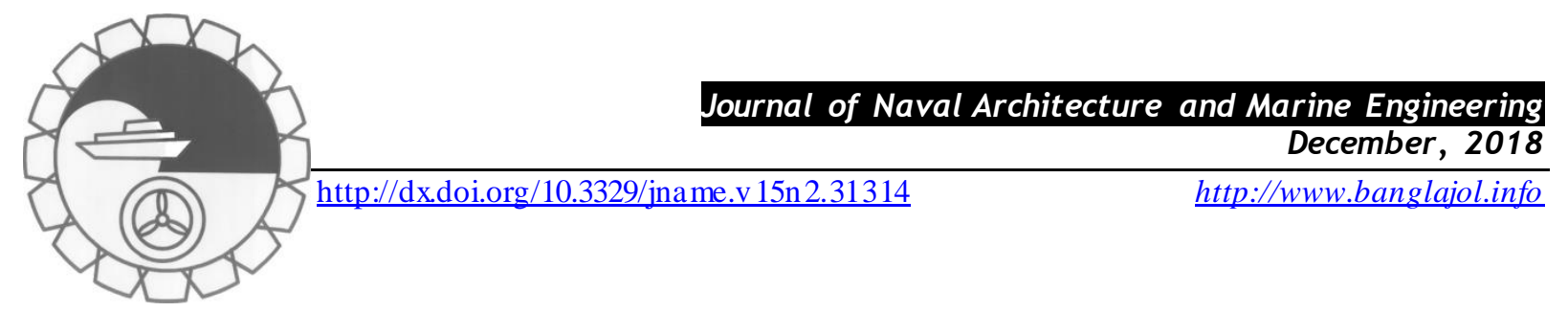

\title{
STUDY OF RAMPED TEMPERATURE INFLUENCE ON MHD CONVECTIVE CHEMICALLY REACTIVE AND ABSORBING FLUID PAST AN EXPONENTIALLY ACCELERATED VERTICAL POROUS PLATE
}

\section{S. Harinath Reddy ${ }^{1}$, M. C. Raju' ${ }^{2}$ and E. Keshava Reddy ${ }^{3}$}

${ }^{1}$ Department of Mathematics, Annamachary a Institute of Technology and Sciences (Autonomous), Rajampet-516126, A.P., India, harinath.singamala@gmail.com

${ }^{2}$ Department of Mathematics, Annamacharya Institute of Technology and Sciences (Autonomous), Rajampet-516126, A.P., India, mcrmaths@yahoo.co.in

${ }^{3}$ Department of Mathematics, JNTUA College of Engineering, Ananthapuramu, A.P., India, keshava_e@ rediffmail.com

\begin{abstract}
:
A systematic study has been performed on MHD convective chemically reactive and absorbing fluid along an exponentially accelerated vertical plate with the impact of Hall current by considering ramped temperature. Laplace transform technique is applied to obtain exact solutions of the non-dimensional governing equations for fluid velocity, temperature and concentration. Based on the above solutions, the expressions for skin friction coefficient, Nussel number and Sherwood number are derived. The consequences of diverse physical parameters on flow quantities are examined thoroughly with graphical representations. The numerical values for skin friction coefficient, rate of heat transfer and rate of mass transfer are recorded and analyzed.
\end{abstract}

Keywords: MHD, free convection, Hall current, chemical reaction, radiation absorption, heat absorption, ramped temperature.

\section{NOMENCLATURE}

$B_{0} \quad$ magnetic field strength

$C_{p} \quad$ specific heat at constant pressure

Gr thermal Grashof number

$k \quad$ thermal conductivity of the fluid.

$k^{*} \quad$ mean absorption coefficient

$K$ permeability of porous medium

$m \quad$ Hall current parameter

$M \quad$ magnetic parameter

$\mathrm{Nr}$ radiation parameter

Sc Schmidt number

$\mathrm{Kr}$ chemical reaction parameter

$\operatorname{Pr} \quad$ Prandtl number

$R \quad$ Radiation absorption parameter

$Q_{0} \quad$ heat absorption parameter

$q_{r} \quad$ radiative heat flux

$t^{*} \quad$ time

$t_{0} \quad$ critical time for rampedness

$t_{1}$ dimensionless critical time for rampedness.

$T \quad$ fluid temperature
$U_{0} \quad$ characteristic velocity

$u \quad$ fluid velocity in $x$-direction

$w \quad$ fluid velocity in $z$-direction

\section{Greek symbols}

$\beta \quad$ coefficient of thermal expansion

$\eta \quad$ non-dimensional space variable

$\sigma^{*} \quad$ Stefan Boltzmann constant

$\sigma \quad$ electrical conductivity

$\rho \quad$ fluid density

$v \quad$ kinematic coefficient of viscosity

$\omega_{\mathrm{e}} \quad$ cyclotron frequency

$\tau_{e} \quad$ electron collision time

$\phi \quad$ heat absorption parameter

\section{Subscripts}

$w \quad$ condition at the wall

$\infty$ free-stream condition/Initial condition at the plate 


\section{Introduction}

Investigation of hydro magnetic free convection flow with heat and mass transfer along porous plates has drawn key attention of researchers, due to its significant role in astrophysics, electronics, aeronautics, geophysics, meteorology, metallurgy, chemical industries and petroleum production. Magneto hydrodynamic (MHD) free convection flow of an electrically conducting fluid through a flooded porous medium has been effectively exploited in crystal formation. Recently the study of Hall current on MHD boundary layer flows with heat transfer has become essential due to its important engineering applications, therefore, researchers are performing their investigations on the applications of MHD towards a strong magnetic field. Generally, in an ionized gas of less density under the existence of strong magnetic field, the conductivity perpendicular to the magnetic field is decreased by free spiral progress of electrons and ions about the magnetic lines of force before suffering collisions. A current which is produced perpendicular to the electromagnetic fields, is known as Hall current. It is mainly utilized in Hall accelerators, electric transformers, refrigeration coils, power generators and pumps, solar physics encountered in the sunspot development, electronic cooling system, cool combustors, fiber and granular insulation, oil extraction, the solar cycle, the structure of magnetic stars, thermal energy storage, flow through filtering devices and porous material regenerative heat exchangers.

Seth et al. (2014) examined and discussed the consequences of Hall current, radiation and rotation parameter on free convective flow past a vertically moving plate in conducting field. Satya Narayan et al.(2014)studied the impact of Hall current and radiation absorption on MHD micro polar fluid in a rotating system. Seth et al. (2016) analyzed the Hall current effects on unsteady hydro magnetic free convection flow past an spontaneously moving plate with the consideration of Newtonian heating. Satyanarayana et al. (2011) analyzed the Hall current effects on MHD flow past a porous plate with free convection. Manglesh et al. (2013) considered and studied magnetohydrodynamic unforced convective flow along porous medium under the existence of Hall current, radiation and thermal diffusion. Saha et al. (2011) discussed thoroughly the impact of Hall current on MHD natural convective flow along perpendicular permeable plate with uniform surface in the presence of heat flux. Pop et al. (1994) considered and analyzed Hall effects on magnetohydrodynamic free convection about a semiinfinite vertical flat plate. Singh et al. (2010) considered and studied transient MHD free convective flow close to a semi- infinite perpendicular wall with the consideration of ramped temperature. Seth et al. (2011) analyzed MHD unforced convection flow past a spontaneously moving plate with ramped wall temperature and radiative heat transfer. Narahari (2012) considered and analyzed transient convective flow between long vertical parallel plates introducing ramped wall temperature at one boundary with the impact of radiation and constant mass transmission. Seth et al. (2016) established unsteady MHD free convection flow of a radiating and heat absorbing fluid along a moving perpendicular plate with varying ramped temperature and Hall effects. Kundu et al. (2014) studied and discussed about the flow features of a conducting fluid closed to an accelerated perpendicular plate flooded in porous medium with the implementation of ramped wall temperature.

In addition to the above studies, chemical reacting MHD free convection flow with radiation effects has acknowledged a mounting importance during the last decades due to its significance in various engineering, geophysical and astrophysical applications, manufacturing of ceramic, packed-bed catalytic reactors, enhanced oil recovery, food processing, underground energy transport, magnetized plasma flow, cooling of nuclear reactors, high speed plasma wind, cosmic jets and stellar system. Srinivas et al. (2014) established the effect of chemical reaction as well as thermal radiation on magnetohydrodynamic flow over an inclined porous extended surface with the consideration of non-uniform heat source/sink: an application to the dynamics of blood flow. Rout et al. (2016) studied the consequences of heat generation and chemical reaction on MHD flow past an upright plate with the consideration of variable temperature. Tripath et al. (2015) considered and discussed about chemical reaction impact on MHD unforced convective surface over an oscillating vertical plate through porous region. Srinivasacharya et al. (2013) considered and examined the influences of chemical reaction and radiation on a non-Newtonian power law fluid saturated with porous medium. Devi et al. (2010) investigated the influences of chemical reaction on MHD flow past a semi-infinite plate with heat and mass transfer. Barik (2014) studied and analyzed chemical reaction and radiation impacts on MHD free convective flow past a spontaneously moving vertical plate with ramped wall temperature as well as ramped wall concentration. Srinivasacharya et al. (2016) studied and discussed about the same effects on mixed convection heat and mass transfer over a vertical plate in power-law fluid saturated with porous medium. Reddy et al. (2016) also considered analyzed these effects on MHD flow along a moving perpendicular porous plate. Prasad et al. (2008) analyzed radiation effects on an unsteady MHD convective heat and mass transfer flow past a semi-infinite 
vertical permeable moving plate embedded in a porous medium. Raju et al. (2016) studied both analytical and numerical study of unsteady MHD free convection flow over an exponentially moving vertical plate in the existence of heat absorption. Hossain et al. (2001) investigated the conseuence of radiation on free convection flow of fluid with variable viscosity from a porous vertical plate. Maboob et al. (2016) examined and discussed the properties of MHD stagnation point flow of nano fluid in porous medium with radiation, viscous dissipation and chemical reaction. Raju and Varma (2014) studied Soret effects due to natural convection in a nonNewtonian fluid flow in porous medium with heat and mass transfer.

From the above studies, it is noticed that, a meager interest is paid towards radiation absorbing fluid. Therefore, the main aim of this investigation is to study analytically the ramped temperature influence on MHD convective chemical reactive and absorbing fluid past an exponentially accelerated vertical plate. The novelty of this study is the consideration of simultaneous occurrence of radiation absorption, heat absorption as well as Hall current effects along with homogeneous chemical reaction. This study though an extension of the work of Seth et al. (2016), it varies in many aspects like radiation absorption, chemical reaction and exponentially accelerated plate etc. Followed by Seth et al. (2016), in this study we have considered $t_{0}$ as critical time for rampedness in place of act eristic time. Due to this reasons, in our study, the interval for rampedness becomes $0<t \leq t_{1}\left(t_{1}\right.$ being the dimensionless critical time for rampedness) where as in the above mentioned research studies, the interval for rampedness is, $0<t \leq 1$ in non-dimensional form. It may be noticed that the physical meaning of critical time for rampedness is the time when plate temperature changes from ramped temperature to uniform temperature.

\section{Formulation of the Problem}

An unsteady free convective flow of a viscous, incompressible, electrically conducting, chemically reacting, optically thick radiating, heat absorbing as well as radiation absorbing fluid past an exponentially accelerated infinite vertical plate in conducting field in the presence of ramped temperature and Hall current is considered. A Cartesian coordinate system $\left(\mathrm{x}^{*}, \mathrm{y}^{*}, \mathrm{z}^{*}\right)$ is chosen in such a way that $\mathrm{x}^{*}$-axis is taken in the upward direction along the vertical plate whereas $\mathrm{y}^{*}$-axis is considered normal to the plane of the plate which is directed into the fluid region and $\mathrm{z}^{*}$ - axis is normal to $\mathrm{x}^{*} \mathrm{y}^{*}$-plane. A uniform transverse magnetic field of strength $\mathrm{B}_{0}$ is applied perpendicular to the plate in a direction parallel to $\mathrm{y}^{*}$-axis. Since the strength of the applied magnetic field is considerably high, therefore the influence of Hall current cannot be neglected. Also the presence of homogeneous chemical reaction is considered. Initially, i.e. at time $t^{*} \leq 0$, both the plate and surrounding fluid are at rest and maintained at uniform temperature $\mathrm{T}_{\infty}^{*}$ and uniform concentration $\mathrm{C}_{\infty}^{*}$. At time $\mathrm{t}^{*}>0$, the plate accelerates exponentially along the $\mathrm{x}^{*}$ direction with a velocity $\mathrm{U}\left(\mathrm{t}^{*}\right)=e^{a^{*} t^{*}}\left(\mathrm{a}^{*}\right.$ being arbitrary constant). At the same time the temperature of the plate raised to $T_{\infty}^{*}+\left(T_{w}^{*}-T_{\infty}^{*}\right)\left(\frac{t^{*}}{t_{0}}\right)$ when $0<t^{*} \leq t_{0}$ and it maintained at uniform temperature $T_{w}^{*}$ when $t^{*}>t_{0}\left(t_{0}\right.$ being critical time for rampedness). And concentration values are maintained at $C_{\infty}^{*}+\left(C_{w}^{*}-C_{\infty}^{*}\right) A t^{*}$, when $t^{*}>0$.

Since the plate is extended infinitely along $x^{*}$ and $z^{*}$ directions, all physical measures except pressure depend on $y^{*}$ and ${ }^{*}$ only. The induced magnetic field generated by movement of the flow is negligible in comparison to applied one. This is reasonable as the magnetic Reynolds number is very minor for partially ionized fluids and liquid metals which are generally used in several industrial processes. The effect of polarization is neglected which corresponds to the case where no energy is added or extracted from the fluid by electrical means. With the above assumptions made, taking Hall parameter into consideration the governing equations for the fluid flow problem, under Boussing approximation, are given below.

$$
\frac{\partial u^{*}}{\partial t^{*}}=v \frac{\partial^{2} u^{*}}{\partial y^{* 2}}-\frac{\sigma B_{0}^{2}}{\rho\left(1+m^{2}\right)}\left(u^{*}+m w^{*}\right)+g \beta\left(T^{*}-T_{\infty}^{*}\right)+g \beta^{*}\left(C^{*}-C_{\infty}^{*}\right)-\frac{v}{k} u^{*}
$$




$$
\begin{aligned}
& \frac{\partial w^{*}}{\partial t^{*}}=v \frac{\partial^{2} w^{*}}{\partial y^{* 2}}+\frac{\sigma B_{0}{ }^{2}}{\rho\left(1+m^{2}\right)}\left(m u^{*}-w^{*}\right)-\frac{v}{k^{*} w^{*}} \\
& \frac{\partial T^{*}}{\partial t^{*}}=\frac{k}{\rho c_{p}} \frac{\partial^{2} T^{*}}{\partial y^{* 2}}-\frac{Q_{0}}{\rho c_{p}}\left(T^{*}-T_{\infty}^{*}\right)-\frac{1}{\rho c_{p}} \frac{\partial q_{r}}{\partial y^{*}}+\frac{Q_{l}}{\rho c_{p}}\left(C^{*}-C_{\infty}^{*}\right) \\
& \frac{\partial C^{*}}{\partial t^{*}}=D \frac{\partial^{2} C^{*}}{\partial{ }^{* 2}}-K_{r}^{*}\left(C^{*}-C_{\infty}^{*}\right)
\end{aligned}
$$

Initial and boundary conditions to be satisfied are as follows

$$
\begin{aligned}
& t^{*} \leq 0: u^{*}=0, w^{*}=0, T^{*}=T_{\infty}^{*}, C^{*}=C_{\infty}^{*}, \text { for all }{ }^{*} \geq 0 \\
& t^{*}>0: u^{*}=e^{a^{*} t^{*}}, w^{*}=0, T^{*}=\left\{\begin{array}{cc}
T_{\infty}^{*}+\left(T_{w^{*}}-T_{\infty}{ }^{*}\right) \frac{t^{*}}{t_{0}} & \text { at }{ }^{*}=0 \text { when } 0<\mathrm{t}^{*} \leq t_{0}, \\
\mathrm{~T}_{w}^{*} & \text { at } \mathrm{y}^{*}=0 \text { when }{ }^{*}>t_{0} .
\end{array}\right. \\
& \text { when } t^{*}>0, \quad C^{*}=C_{\infty}^{*}+\left(C_{w}^{*}-C_{\infty}^{*}\right) A t^{*} \text { at }{ }^{*}=0 \\
& t^{*}>0: \quad u^{*} \rightarrow 0, \quad w^{*} \rightarrow 0, \quad T^{*} \rightarrow T_{\infty}{ }^{*}, \quad C^{*} \rightarrow C_{\infty}{ }^{*}, \quad \text { as } \quad{ }^{*} \rightarrow \infty
\end{aligned}
$$

For an optically low thick gray fluid, the radiative heat flux $q_{r}$ is approximated by Roseland approximation which is given as

$$
q_{r}=-\frac{4 \sigma^{*}}{3 k^{*}} \frac{\partial T^{* 4}}{\partial y^{*}}
$$

It is assumed that the temperature difference between fluid in the boundary layer region and free-stream is very small so that is $T^{* 4}$ being expressed as a linear function of temperature $T^{*}$. Expanding $T^{* 4}$ in Taylor series about $T_{\infty}^{*}$ and neglecting second and higher order terms, we get

$T^{* 4}=4 T_{\infty}^{* 3} T^{*}-3 T_{\infty}^{* 4}$

Using equations (8) and (9) in (3) we get

$$
\frac{\partial T^{*}}{\partial t^{*}}=\frac{k}{p c_{p}}\left(1+\frac{16 \sigma^{*} T_{\infty}^{* 3}}{3 k k^{*}}\right) \frac{\partial^{2} T^{*}}{\partial y^{* 2}}-\frac{Q_{0}}{\rho c_{p}}\left(T^{*}-T_{\infty}^{*}\right)+\frac{Q_{l}}{\rho c_{p}}\left(C^{*}-C_{\infty}^{*}\right)
$$

We introduce the following non dimensional quantities and flow parameters to present (1), (2) and (10) along with initial and boundary conditions (5)-(7) in non-dimensional form

$$
\begin{aligned}
& \eta=\frac{U_{0} y^{*}}{v}, \quad t=\frac{U_{0} t^{*}}{v}, \quad u=\frac{u^{*}}{U_{0}}, \quad w=\frac{w^{*}}{U_{0}}, \quad T=\frac{\left(T^{*}-T^{*}{ }_{\infty}\right)}{\left(T_{w}^{*}-T_{\infty}^{*}\right)}, \quad S_{c}=\frac{v U_{0}{ }^{2}}{D U_{0}^{4}} \\
& G_{r}=\frac{v g \beta\left(T^{*}{ }^{*}-T_{\infty}^{*}\right)}{U_{0}{ }^{3}}, \quad C=\frac{\left(C^{*}-C_{\infty}^{*}\right)}{\left(C_{w}^{*}-C_{\infty}^{*}\right)}, \quad G_{c}=\frac{v g \beta^{*}\left(C^{*}{ }^{*}-C_{\infty}^{*}\right)}{U_{0}{ }^{3}} \\
& M^{2}=\frac{\sigma B_{0}^{2}{ }_{0}^{v}}{\rho U_{0}{ }^{2}}, \quad N_{r}=\frac{16 \sigma^{*} T^{* 3}}{3 k k^{*}}, \quad t_{1}=\frac{U_{0}{ }^{2} t_{0}}{v}, \quad K=\frac{k^{*} U_{0}^{2}}{v^{2}},
\end{aligned}
$$


$P_{r}=\frac{\sigma v c_{p}}{k}, \quad \phi=\frac{Q_{0} v}{\sigma c_{p} U_{0}^{2}}, \quad K_{r}=\frac{K_{r}^{*} v}{U_{0}^{2}}, R=\frac{Q_{l} v\left(C_{w}-C_{\infty}\right)}{\rho c_{p} U_{0}^{2}\left(T_{w}-T_{\infty}\right)}, A=\frac{U_{0}^{2}}{v}$

Making use of equation (11), equations (1), (2), (4) and (10), in non-dimensional form, reduce to

$\frac{\partial F}{\partial t}+\frac{M^{2}(1-i m) F}{\left(1+m^{2}\right)}=\frac{\partial^{2} F}{\partial \eta^{2}}+G_{r} T+G_{C} C-\frac{1}{K} F$

$\frac{\partial T}{\partial t}=\frac{\left(1+N_{r}\right)}{P_{r}} \frac{\partial^{2} T}{\partial \eta^{2}}-\phi T+R C$

$\frac{\partial C}{\partial t}=\frac{1}{S_{c}} \frac{\partial^{2} C}{\partial \eta^{2}}-K_{r} C$

where $F(y, t)=u(y, t)+i w(y, t)$.

\section{Solution of the Problem}

Initial and boundary conditions (5) to (7), in non-dimensional form, are given by

$t \leq 0: F=0, \quad T=0, \quad C=0 \quad$ for all $\eta \geq 0$

$t>0: F=e^{a t}, \quad T=\left\{\begin{array}{ll}\frac{t}{t_{1}} & \text { at } \eta=0 \text { when } 0<\mathrm{t} \leq t_{1}, \\ 1 & \text { at } \eta=0 \text { when } \mathrm{t}>t_{1} .\end{array}\right.$ when $t^{*}>0, C=t$ at $\eta=0$

$t>0: F \rightarrow 0, \quad T \rightarrow 0, \quad C \rightarrow 0 \quad$ as $\eta \rightarrow \infty$

where $a=\frac{a * v}{U_{0}^{3}}$ is non-dimensional constant.

Using Laplace transform technique, first the solution for fluid temperature $T(\eta, t)$ is obtained by solving equation (13) and the solution for fluid concentration $C(\eta, t)$ is obtained by solving equation (14), then using this solution in equation (12), solution for fluid velocity $F(\eta, t)$ is obtained. The exact solutions obtained are as follows:

$$
\begin{aligned}
& C(\eta, t)=\left(\frac{t}{2}-\frac{\eta \sqrt{s_{c}}}{4 \sqrt{K_{r}}}\right) e^{-y \sqrt{S_{c} K_{r}}} \operatorname{erfc}\left(\frac{\eta \sqrt{s_{c}}}{2 \sqrt{t}}-\sqrt{K_{r} t}\right) \\
&+\left(\frac{t}{2}+\frac{\eta \sqrt{s_{c}}}{4 \sqrt{K_{r}}}\right) e^{y \sqrt{S_{c} K_{r}}} \operatorname{erfc}\left(\frac{\eta \sqrt{s_{c}}}{2 \sqrt{t}}+\sqrt{K_{r} t}\right) \\
& T(\eta, t)=\frac{1}{t_{1}}\left(T_{1}(\eta, t)-H\left(t-t_{1}\right) T_{1}\left(\eta, t-t_{1}\right)\right)-A_{5} T_{1}(\eta, t)+A_{4} T_{3}(\eta, t)+ \\
& A_{6} T_{5}(\eta, t)-A_{4} T_{6}(\eta, t)-A_{6} T_{7}(\eta, t)+A_{5} C(\eta, t)
\end{aligned}
$$


S. H. Reddy, M. C. Raju, E. K. Reddy/Journal of NavalArchitectureandMarine Engineering, 15(2018) 107-125

$$
\begin{aligned}
F(\eta, t) & =F_{1}(\eta, t)-A_{38} F_{2}(\eta, t)-A_{39} F_{3}(\eta, t)-A_{40}\left[F_{4}(\eta, t)-F_{9}(\eta, t)\right]-A_{41} F_{5}(\eta, t) \\
& -A_{42}\left[F_{6}(\eta, t)-F_{13}(\eta, t)\right]+A_{12} H\left(t-t_{1}\right) F_{2}\left(\eta, t-t_{1}\right)+A_{11} H\left(t-t_{1}\right) F_{3}\left(\eta, t-t_{1}\right) \\
& +A_{10} H\left(t-t_{1}\right) F_{4}\left(\eta, t-t_{1}\right)+A_{43} F_{7}(\eta, t)+A_{44} F_{8}(\eta, t)+A_{21} F_{10}(\eta, t) \\
& -A_{12} H\left(t-t_{1}\right) F_{7}\left(\eta, t-t_{1}\right)-A_{11} H\left(t-t_{1}\right) F_{8}\left(\eta, t-t_{1}\right)-A_{10} H\left(t-t_{1}\right) F_{9}\left(\eta, t-t_{1}\right) \\
& +A_{45} F_{11}(\eta, t)+A_{46} F_{12}(\eta, t)+A_{32} F_{14}(\eta, t)
\end{aligned}
$$

Where

$$
\begin{aligned}
& T_{1}(\eta, t)=\left(\frac{t}{2}-\alpha_{1}\right) e^{-\alpha_{4}} \operatorname{erfc}\left(\alpha_{2}-\alpha_{3}\right)+\left(\frac{t}{2}+\alpha_{1}\right) e^{\alpha_{4}} \operatorname{erfc}\left(\alpha_{2}+\alpha_{3}\right) \\
& T_{3}(\eta, t)=(1 / 2)\left\{e^{-\alpha_{5}} \operatorname{erfc}\left(\alpha_{6}-\alpha_{7}\right)+e^{\alpha_{5}} \operatorname{erfc}\left(\alpha_{6}+\alpha_{7}\right)\right\} \\
& T_{4}(\eta, t)=\left(\frac{t}{2}-\alpha_{8}\right) e^{-\alpha_{5}} \operatorname{erfc}\left(\alpha_{6}-\alpha_{7}\right)+\left(\frac{t}{2}+\alpha_{8}\right) e^{\alpha_{5}} \operatorname{erfc}\left(\alpha_{6}+\alpha_{7}\right) \\
& T_{5}(\eta, t)=\left(\frac{e^{-A_{3} t}}{2}\right)\left\{e^{-\eta \sqrt{S c\left(K r-A_{3}\right)}} \operatorname{erfc}\left(\alpha_{6}-\sqrt{\left(K_{r}-A_{3}\right) t}\right)+e^{\eta \sqrt{S_{c}\left(K_{r}-A_{3}\right)}} \operatorname{erfc}\left(\alpha_{6}+\sqrt{\left(K_{r}-A_{3}\right) t}\right)\right\} \\
& T_{6}(\eta, t)=\left(\frac{1}{2}\right)\left\{e^{-\alpha_{4}} \operatorname{erfc}\left(\alpha_{2}-\alpha_{3}\right)+e^{\alpha_{4}} \operatorname{erfc}\left(\alpha_{2}+\alpha_{3}\right)\right\} \\
& T_{7}(\eta, t)=\left(\frac{e^{-A_{3} t}}{2}\right)\left\{e^{-\eta \sqrt{\frac{\phi-A_{3}}{A_{1}}}} \operatorname{erfc}\left(\alpha_{2}-\sqrt{\left(\phi-A_{3}\right) t}\right)+e^{\eta \sqrt{\frac{\phi-A_{3}}{A_{1}}}} \operatorname{erfc}\left(\alpha_{2}+\sqrt{\left(\phi-A_{3}\right) t}\right)\right\} \\
& F_{1}(\eta, t)=\frac{e^{a t}}{2}\left[e^{-\eta \sqrt{a-A_{7}}} \operatorname{erfc}\left(\alpha_{9}-\sqrt{\left(a-A_{7}\right) t}\right)+e^{\eta \sqrt{a-A_{7}}} \operatorname{erfc}\left(\alpha_{9}+\sqrt{\left(a-A_{7}\right) t}\right)\right] \\
& F_{2}(\eta, t)=\frac{1}{2}\left[e^{-\eta \sqrt{-A_{7}}} \operatorname{erfc}\left(\alpha_{9}-\sqrt{\left(-A_{7}\right)} t\right)+e^{\eta \sqrt{-A_{7}}} \operatorname{erfc}\left(\alpha_{9}+\sqrt{\left(-A_{7}\right)} t\right)\right] \\
& F_{3}(\eta, t)=\left(\frac{t}{2}-\frac{\eta}{4 \sqrt{-A_{7}}}\right) e^{-\eta \sqrt{-A_{7}}} \operatorname{erfc}\left(\alpha_{9}-\sqrt{\left(-A_{7}\right) t}\right)+\left(\frac{t}{2}+\frac{\eta}{4 \sqrt{-A_{7}}}\right) e^{\eta \sqrt{-A_{7}}} \operatorname{erfc}\left(\alpha_{9}+\sqrt{\left(-A_{7}\right) t}\right) \\
& F_{4}(\eta, t)=\frac{e^{-A_{9} t}}{2}\left[e^{-\eta \sqrt{-A_{9}-A_{7}}} \operatorname{erfc}\left(\alpha_{9}-\sqrt{\left(-A_{9}-A_{7}\right) t}\right)+e^{\eta \sqrt{-A_{9}-A_{7}}} \operatorname{erfc}\left(\alpha_{9}+\sqrt{\left(-A_{9}-A_{7}\right) t}\right)\right] \\
& F_{5}(\eta, t)=\frac{e^{-A_{3} t}}{2}\left[e^{-\eta \sqrt{-A_{3}-A_{7}}} \operatorname{erfc}\left(\alpha_{9}-\sqrt{\left(-A_{3}-A_{7}\right)} t\right)+e^{\eta \sqrt{-A_{3}-A_{7}}} \operatorname{erfc}\left(\alpha_{9}+\sqrt{\left(-A_{3}-A_{7}\right) t}\right)\right] \\
& F_{6}(\eta, t)=\frac{e^{-A_{24} t}}{2}\left[e^{-\eta \sqrt{-A_{24}-A_{7}}} \operatorname{erfc}\left(\alpha_{9}-\sqrt{\left(-A_{24}-A_{7}\right) t}\right)+e^{\eta \sqrt{-A_{24}-A_{7}}} \operatorname{erfc}\left(\alpha_{9}+\sqrt{\left(-A_{24}-A_{7}\right) t}\right)\right] \\
& F_{7}(\eta, t)=T_{6}(\eta, t) \quad F_{8}(\eta, t)=T_{1}(\eta, t)
\end{aligned}
$$

Study of ramped temperature influence on MHD convective chemically reactive and absorbingfluidpast an exponentially.......

112 


$$
\begin{aligned}
& F_{9}(\eta, t)=\left(\frac{e^{-A_{9} t}}{2}\right)\left\{e^{-\eta \sqrt{\frac{\phi-A_{9}}{A_{1}}}} \operatorname{erfc}\left(\alpha_{2}-\sqrt{\left(\phi-A_{9}\right) t}\right)+e^{\eta \sqrt{\frac{\phi-A_{9}}{A_{1}}}} \operatorname{erfc}\left(\alpha_{2}+\sqrt{\left(\phi-A_{9}\right)} t\right)\right\} \\
& F_{10}(\eta, t)=T_{7}(\eta, t), F_{11}(\eta, t)=T_{3}(\eta, t) F_{12}(\eta, t)=T_{4}(\eta, t) \\
& F_{13}(\eta, t)=\left(\frac{e^{-A_{24} t}}{2}\right)\left\{e^{-\eta \sqrt{S c\left(K r-A_{24}\right)}} \operatorname{erfc}\left(\alpha_{6}-\sqrt{\left(K_{r}-A_{24}\right) t}\right)+e^{\eta \sqrt{S_{c}\left(K_{r}-A_{24}\right)}} \operatorname{erfc}\left(\alpha_{6}+\sqrt{\left(K_{r}-A_{3}\right) t}\right)\right\} \\
& F_{14}(\eta, t)=T_{5}(\eta, t)
\end{aligned}
$$

Where

$$
\begin{aligned}
& A_{1}=\frac{1+N_{r}}{\operatorname{Pr}}, A_{2}=\frac{-R}{\left(S_{c} A_{1}-1\right)}, A_{3}=\frac{\left(S_{c} K_{r} A_{1}-\phi\right)}{\left(S_{c} A_{1}-1\right)}, A_{4}=\frac{-A_{2}}{A_{3}^{2}}, A_{5}=\frac{A_{2}}{A_{3}}, A_{6}=-A_{4} \\
& A_{7}=\frac{M^{2}}{1+m^{2}}(m i-1)-\frac{1}{K}, A_{8}=\frac{-G r}{t_{1}\left(1-A_{1}\right)}, A_{9}=\frac{\phi+A_{1} A_{7}}{1-A_{1}}, A_{10}=\frac{A_{8}}{A_{9}^{2}}, A_{11}=\frac{A_{8}}{A_{9}}, A_{12}=-A_{10} \\
& A_{13}=\frac{G r A_{4} A_{1}}{\left(1-A_{1}\right)}, A_{14}=\frac{A_{13}}{A_{9}}, A_{15}=-A_{14}, A_{16}=\frac{G r A_{5} A_{1}}{\left(1-A_{1}\right)}, A_{17}=\frac{-A_{16}}{A_{9}^{2}}, A_{18}=\frac{A_{16}}{A_{9}}, A_{19}=-A_{17} \\
& A_{20}=\frac{G r A_{6} A_{1}}{\left(1-A_{1}\right)}, A_{21}=\frac{A_{20}}{A_{9}-A_{3}}, A_{22}=\frac{A_{20}}{A_{3}-A_{9}}, A_{23}=-\frac{G r A_{4}}{\left(S_{c}-1\right)}, A_{24}=\frac{S_{c} K_{r}+A_{7}}{\left(S_{c}-1\right)}, A_{25}=\frac{A_{23}}{A_{24}} \\
& A_{26}=-A_{25}, \quad A_{27}=-\frac{G r A_{5}}{\left(S_{c}-1\right)}, A_{28}=-\frac{A_{27}}{A_{24}^{2}}, A_{29}=\frac{A_{27}}{A_{24}}, A_{30}=-A_{28}, \quad A_{31}=-\frac{G r A_{6}}{\left(S_{c}-1\right)} \\
& A_{32}=\frac{A_{31}}{A_{24}-A_{3}}, \quad A_{33}=\frac{A_{31}}{A_{3}-A_{24}}, A_{34}=\frac{G_{c}}{\left(S_{c}-1\right)}, \quad A_{35}=-\frac{A_{34}}{A_{24}^{2}}, A_{36}=\frac{A_{34}}{A_{24}}, A_{37}=-A_{35} \\
& A_{38}=A_{12}+A_{14}+A_{17}+A_{25}+A_{28}-A_{35}, A_{39}=A_{11}+A_{18}+A_{29}-A_{36}, \quad A_{40}=A_{10}+A_{15}+A_{19}+A_{22} \\
& A_{41}=A_{21}+A_{32}, \quad A_{42}=A_{26}+A_{30}+A_{33}-A_{37}, A_{43}=A_{12}+A_{14}+A_{17}, \\
& A_{44}=A_{11}+A_{18}, \quad A_{45}=A_{25}+A_{28}-A_{35}, \quad A_{46}=A_{29}-A_{36} \\
& \alpha_{1}=\frac{\eta}{4 \sqrt{A_{1} \phi}}, \alpha_{2}=\frac{\eta}{2 \sqrt{A_{1}} t}, \alpha_{3}=\sqrt{\phi t}, \alpha_{4}=\eta \sqrt{\frac{\phi}{A_{1}}}, \alpha_{5}=\eta \sqrt{S_{c} K_{r}}, \alpha_{6}=\frac{\eta \sqrt{S_{c}}}{2 \sqrt{t}}, \alpha_{7}=\sqrt{K_{r} t} \\
& \alpha_{8}=\frac{\eta \sqrt{S_{c}}}{4 \sqrt{K_{r}}}, \alpha_{9}=\frac{\eta}{2 \sqrt{t}}
\end{aligned}
$$

\subsection{Mass transfer coefficient}

Another important physical quantity is the mass transfer coefficient, i.e. the Sherwood number which is in non dimensional form is given by

$$
S h=-\left(\frac{\partial C}{\partial \eta}\right)_{\eta=0}=\left(\frac{\sqrt{S_{c}}}{2 \sqrt{K_{r}}}+t \sqrt{S_{c} K_{r}}\right) \operatorname{erfc} \sqrt{K_{r} t}-\frac{\sqrt{S_{c}}}{2 \sqrt{K_{r}}}-t \sqrt{S_{c} K_{r}}-\frac{\sqrt{t S_{c}}}{\sqrt{\pi}} e^{-K_{r} t}
$$




\subsection{Heat transfer coefficient}

Expression for rate of heat transfer at the plate i.e.the rate of heat transfer in terms of Nusselt number is given by

$N u=-\left(\frac{\partial T}{\partial \eta}\right)_{\eta=0}=\left(\frac{1}{t_{1}}\right)\left[N_{1}-H\left(t-t_{1}\right) N_{2}\right]+A_{4}\left(N_{3}-N_{6}\right)+A_{6}\left(N_{5}-N_{7}\right)+A_{5}\left(N_{4}-N_{1}\right)$

\subsection{Skin friction coefficient}

The skin friction at the plate in non-dimensional form is given by

$$
\begin{aligned}
\tau=-\left(\frac{\partial F}{\partial \eta}\right)_{\eta=0}=f_{1}-A_{38} f_{2}-A_{39} f_{3}-A_{40} f_{4}-A_{41} f_{5}-A_{42} f_{6}+A_{12} H\left(t-t_{1}\right) f_{21}+A_{11} H\left(t-t_{1}\right) f_{31} \\
+A_{10} H\left(t-t_{1}\right) f_{41}+A_{43} f_{7}+A_{44} f_{8}+A_{40} f_{9}+A_{21} f_{10}-A_{12} H\left(t-t_{1}\right) f_{71}- \\
A_{11} H\left(t-t_{1}\right) f_{81}-A_{10} H\left(t-t_{1}\right) f_{91}+A_{45} f_{10}+A_{46} f_{12}+A_{42} f_{13}+A_{32} f_{14}
\end{aligned}
$$

where

$$
\begin{aligned}
& N_{1}=\left(t \sqrt{\frac{\phi}{A_{1}}}+\frac{1}{2 \sqrt{\phi A_{1}}}\right) \operatorname{erfc}(\sqrt{\phi t})-\frac{1}{2 \sqrt{\phi A_{1}}}-t \sqrt{\frac{\phi}{A_{1}}}-e^{-\phi t} \sqrt{\frac{t}{\pi A_{1}}} \\
& N_{2}=\left(\left(t-t_{1}\right) \sqrt{\frac{\phi}{A_{1}}}+\frac{1}{2 \sqrt{\phi A_{1}}}\right) \operatorname{erfc}\left(\sqrt{\phi\left(t-t_{1}\right)}\right)-\frac{1}{2 \sqrt{\phi A_{1}}}-\left(t-t_{1}\right) \sqrt{\frac{\phi}{A_{1}}}-e^{-\phi\left(t-t_{1}\right)} \sqrt{\frac{\left(t-t_{1}\right)}{\pi A_{1}}} \\
& N_{3}=\left(\sqrt{S_{c} K_{r}}\right) \operatorname{erfc} \sqrt{K_{r} t}-e^{-K_{r} t} \sqrt{\frac{S_{c}}{\pi t}}-\sqrt{S_{c} K_{r}} \\
& N_{4}=\left(2 \sqrt{\frac{S c}{K r}}+t \sqrt{S_{c} K_{r}}\right) \operatorname{erfc} \sqrt{K_{r} t}-\frac{\sqrt{S_{c}}}{2 \sqrt{K_{r}}}-t \sqrt{S_{c} K_{r}}-e^{-K_{r} t} \sqrt{\frac{t S_{c}}{\pi}} \\
& N_{5}=e^{-A_{3} t}\left\{\operatorname{erfc}\left(\sqrt{\left(K_{r}-A_{3}\right) t}\right) \sqrt{S c\left(K_{r}-A_{3}\right)}-e^{-\left(K_{r}-A_{3}\right) t} \sqrt{\frac{S_{c}}{\pi t}}-\sqrt{S c\left(K_{r}-A_{3}\right)}\right\} \\
& N_{6}=\sqrt{\frac{\phi}{A_{1}}} \operatorname{erfc} \sqrt{\phi t}-\sqrt{\frac{\phi}{A_{1}}}-\frac{1}{\sqrt{\pi A_{1} t}} e^{-\phi t}
\end{aligned}
$$

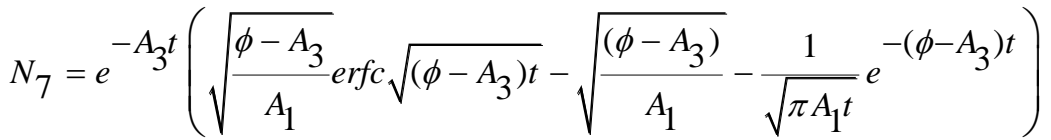

$$
\begin{aligned}
& f_{1}=e^{a t}\left(\sqrt{a-A_{7}} \operatorname{erfc}\left(\sqrt{\left(a-A_{7}\right) t}\right)-\sqrt{a-A_{7}}-\frac{e^{-\left(a-A_{7}\right) t}}{\sqrt{\pi t}}\right) \\
& f_{2}=\left(\sqrt{-A_{7}} \operatorname{erfc}\left(\sqrt{\left(-A_{7}\right) t}\right)-\sqrt{-A_{7}}-\frac{e^{\left(A_{7}\right) t}}{\sqrt{\pi t}}\right) \\
& f_{3}=\left(t \sqrt{-A_{7}}+\frac{1}{2 \sqrt{-A_{7}}}\right) \operatorname{erfc} \sqrt{\left(-A_{7}\right) t}-\sqrt{\frac{t}{\pi}} e^{A_{7} t}-t \sqrt{-A_{7}}-\frac{1}{2 \sqrt{-A_{7}}} \\
& f_{4}=e^{-A_{9} t}\left(\sqrt{-A_{9}-A_{7}} \operatorname{erfc}\left(\sqrt{\left(-A_{9}-A_{7}\right) t}\right)-\sqrt{-A_{9}-A_{7}}-\frac{e^{-\left(-A_{9}-A_{7}\right) t}}{\sqrt{\pi t}}\right)
\end{aligned}
$$




$$
\begin{aligned}
& f_{5}=e^{-A_{3} t}\left(\sqrt{-A_{3}-A_{7}} \operatorname{erfc}\left(\sqrt{\left(-A_{3}-A_{7}\right) t}\right)-\sqrt{-A_{3}-A_{7}}-\frac{e^{-\left(-A_{3}-A_{7}\right) t}}{\sqrt{\pi t}}\right) \\
& f_{6}=e^{-A_{24} t}\left(\sqrt{-A_{24}-A_{7}} \operatorname{erfc}\left(\sqrt{\left(-A_{24}-A_{7}\right) t}\right)-\sqrt{-A_{24}-A_{7}}-\frac{e^{-\left(-A_{24}-A_{7}\right) t}}{\sqrt{\pi t}}\right) \\
& f_{21}=\left(\sqrt{-A_{7}} \operatorname{erfc}\left(\sqrt{\left(-A_{7}\right)\left(t-t_{1}\right)}\right)-\sqrt{-A_{7}}-\frac{e^{A_{7}\left(t-t_{1}\right)}}{\sqrt{\pi\left(t-t_{1}\right)}}\right) \\
& f_{31}=\left(\left(t-t_{1}\right) \sqrt{-A_{7}}+\frac{1}{2 \sqrt{-A_{7}}}\right) \operatorname{erfc} \sqrt{\left(-A_{7}\right)\left(t-t_{1}\right)}-\sqrt{\frac{\left(t-t_{1}\right)}{\pi}} e^{A_{7}\left(t-t_{1}\right)}-\left(t-t_{1}\right) \sqrt{-A_{7}}-\frac{1}{2 \sqrt{-A_{7}}} \\
& f_{41}=e^{-A_{24} t}\left(\sqrt{-A_{24}-A_{7}} \operatorname{erfc}\left(\sqrt{\left(-A_{24}-A_{7}\right) t}\right)-\sqrt{-A_{24}-A_{7}}-\frac{e^{-\left(-A_{24}-A_{7}\right) t}}{\sqrt{\pi t}}\right) \\
& f_{7}=\sqrt{\frac{\phi}{A_{1}}} \operatorname{erfc} \sqrt{\phi t}-\sqrt{\frac{\phi}{A_{1}}}-\frac{1}{\sqrt{\pi A_{1} t}} e^{-\phi t}, f_{8}=\left(t \sqrt{\frac{\phi}{A_{1}}}+\frac{1}{2 \sqrt{\phi A_{1}}}\right) \operatorname{erfc}(\sqrt{\phi t})-\frac{1}{2 \sqrt{\phi A_{1}}}-t \sqrt{\frac{\phi}{A_{1}}}-e^{-\phi t} \sqrt{\frac{t}{\pi A_{1}}} \\
& f_{9}=e^{-A_{9} t}\left(\sqrt{\frac{\left(\phi-A_{9}\right)}{A_{1}}} \operatorname{erfc} \sqrt{\left(\phi-A_{9}\right) t}-\sqrt{\frac{\left(\phi-A_{9}\right)}{A_{1}}}-\frac{1}{\sqrt{\pi A_{1} t}} e^{-\left(\phi-A_{9}\right) t}\right)
\end{aligned}
$$

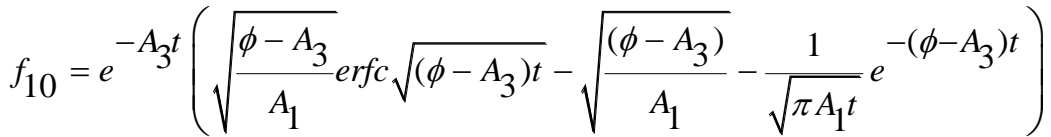

$$
\begin{aligned}
& f_{71}=\sqrt{\frac{\phi}{A_{1}}} \operatorname{erfc} \sqrt{\phi\left(t-t_{1}\right)}-\sqrt{\frac{\phi}{A_{1}}}-\frac{1}{\sqrt{\pi A_{1}\left(t-t_{1}\right)}} e^{-\phi\left(t-t_{1}\right)} \\
& f_{81}=\left(\left(t-t_{1}\right) \sqrt{\frac{\phi}{A_{1}}}+\frac{1}{2 \sqrt{\phi A_{1}}}\right) \operatorname{erfc}\left(\sqrt{\phi\left(t-t_{1}\right)}\right)-\frac{1}{2 \sqrt{\phi A_{1}}}-\left(t-t_{1}\right) \sqrt{\frac{\phi}{A_{1}}}-e^{-\phi\left(t-t_{1}\right)} \sqrt{\frac{\left(t-t_{1}\right)}{\pi A_{1}}} \\
& f_{91}=e^{-A_{9}\left(t-t_{1}\right)}\left(\sqrt{\frac{\left(\phi-A_{9}\right)}{A_{1}}} \operatorname{erfc} \sqrt{\left(\phi-A_{9}\right)\left(t-t_{1}\right)}-\sqrt{\frac{\left(\phi-A_{9}\right)}{A_{1}}}-\frac{1}{\sqrt{\pi A_{1}\left(t-t_{1}\right)}} e^{-\left(\phi-A_{9}\right)\left(t-t_{1}\right)}\right) \\
& f_{11}=\left(\sqrt{S_{c} K_{r}}\right) \operatorname{erfc} \sqrt{K_{r} t}-e^{-K_{r} t} \sqrt{\frac{S_{c}}{\pi t}}-\sqrt{S_{c} K_{r}} \\
& f_{12}=\left(2 \sqrt{\frac{S c}{K r}}+t \sqrt{S_{c} K_{r}}\right) \operatorname{erfc} \sqrt{K_{r} t}-\frac{\sqrt{S_{c}}}{2 \sqrt{K_{r}}}-t \sqrt{S_{c} K_{r}}-e^{-K_{r} t \sqrt{\frac{t S_{c}}{\pi}}} \\
& f_{13}=e^{-A_{24} t}\left\{\operatorname{erfc}\left(\sqrt{\left(K_{r}-A_{24}\right) t}\right) \sqrt{S c\left(K_{r}-A_{24}\right)}-e^{-\left(K_{r}-A_{24}\right) t} \sqrt{\frac{S_{c}}{\pi t}}-\sqrt{S c\left(K_{r}-A_{24}\right)}\right\} \\
& f_{14}=e^{-A_{3} t}\left\{\operatorname{erfc}\left(\sqrt{\left(K_{r}-A_{3}\right) t}\right) \sqrt{S c\left(K_{r}-A_{3}\right)}-e^{-\left(K_{r}-A_{3}\right) t} \sqrt{\frac{S_{c}}{\pi t}}-\sqrt{S c\left(K_{r}-A_{3}\right)}\right\}
\end{aligned}
$$




\section{Validation of the Result}

To validate the present methodology, a comparis on has been performed, by considering the temperature profiles under the influence of thermal radiation. The current results are compared with the results of Seth et al. [2016] in the absence of radiation absorption, mass diffusion and chemical reaction. An excellent agreement is noticed in this comparison which validates the present Laplace technique.

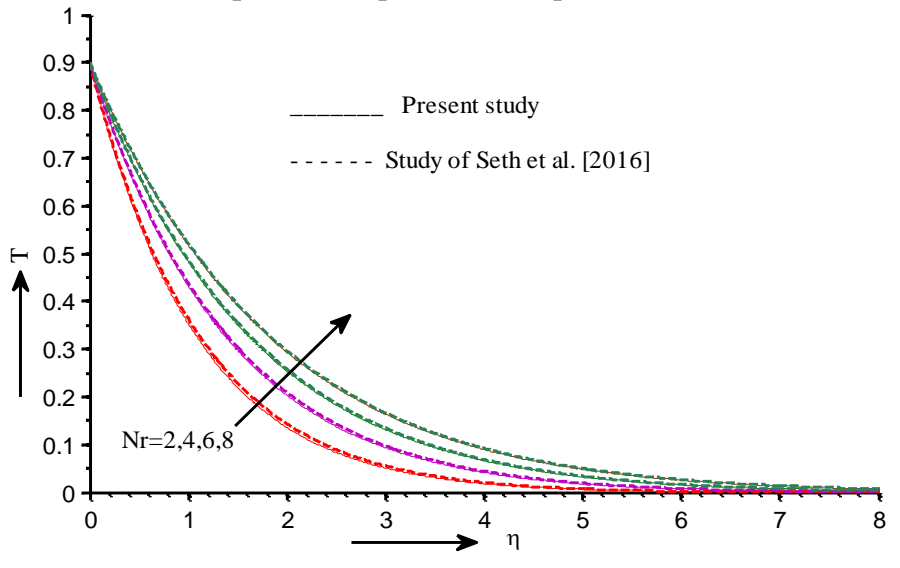

Fig. 1: Temperature profiles under the influence of thermal radiation.

\section{Results and Discussion}

Fig. 2(a) and Fig. 2(b) depict the consequence of Gr on the primary and secondary fluid velocities. From these two figures, it is evident that, the flow velocity enhances with increasing values of Gr. The similar nature of the primary and secondary velocities of the fluid are noticed in the case of Gc, which are evident in the Fig. 3(a) and Fig. 3(b). This is due to relative strength of thermal buoyancy force to viscous force. Since, fluid flow in this problem is induced due to free convection arising as a result of thermal buoyancy force; therefore, thermal buoyancy force will obviously tend to accelerate fluid flow in both the primary and secondary flow directions throughout the boundary layer region.

Fig. 4(a) and Fig. 4(b) illustrate the effect of $\mathrm{Nr}$ on primary and secondary fluid velocities. From these two figures, it is noticed that, the velocity of the fluid decreases with an increasing values of $\mathrm{Nr}$. This happens due to the retarding nature of thermal radiation. Fig. 5(a) and Fig. 5(b), exhibit the effect of radiation absorption on the primary and secondary velocity of the fluid. It is observed that $u$ and $w$ increases on increasing $R$. The radiation absorption accelerates the flow and hence the velocity grows.

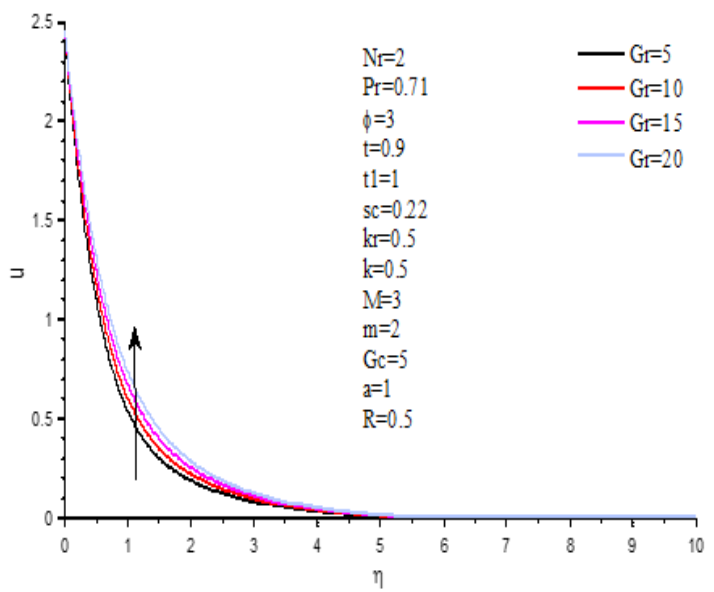

Fig. 2(a): Effect of Gr on u.

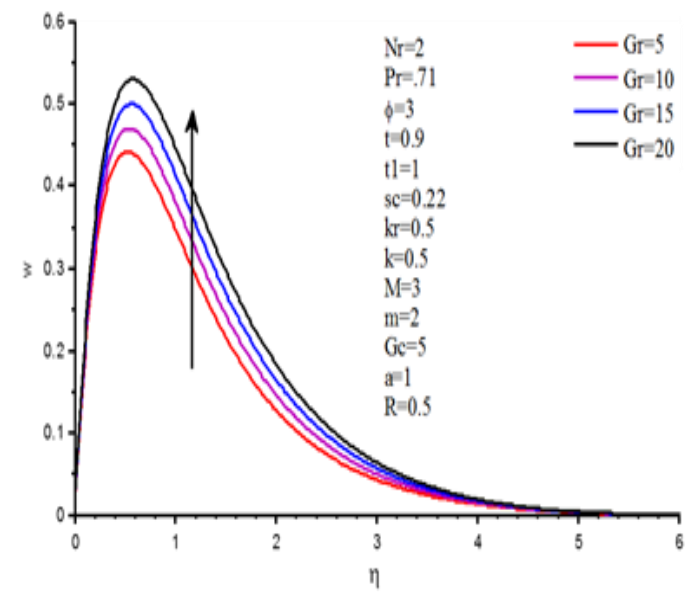

Fig. 2(b): Effect of Gr on w 


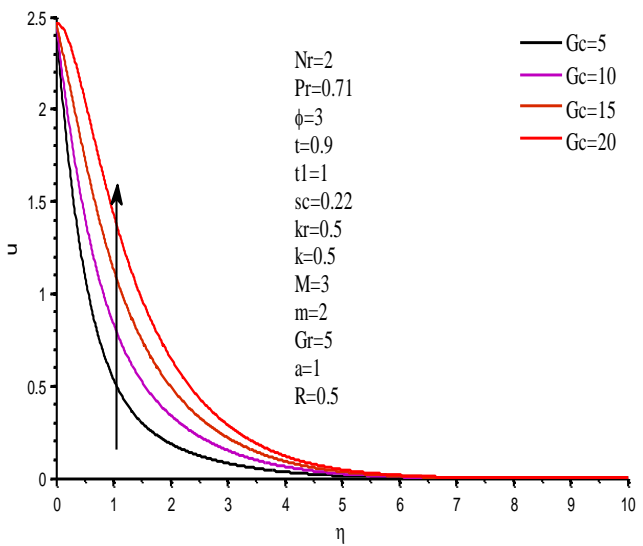

Fig. 3(a): Effect of Gc on u.

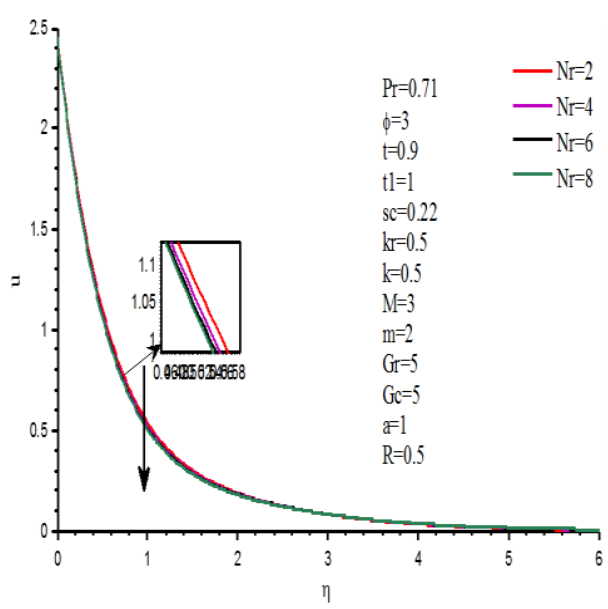

Fig. 4(a): Effect of $\mathrm{Nr}$ on $\mathrm{u}$.

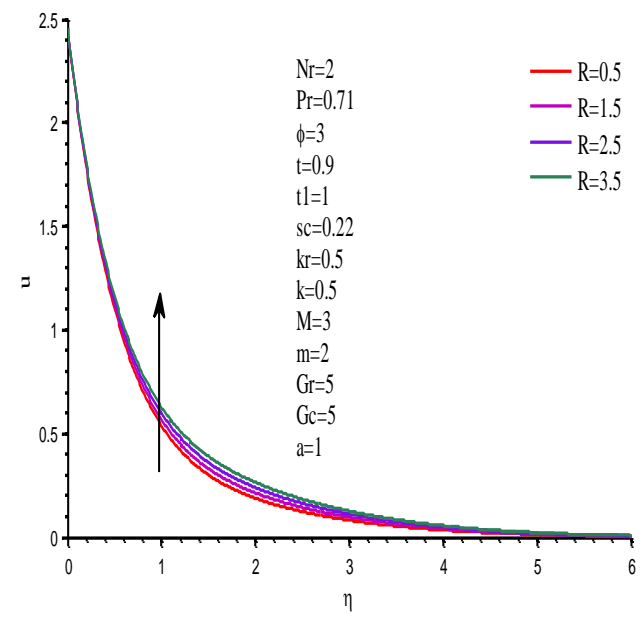

Fig. 5(a): Effect of R on u.

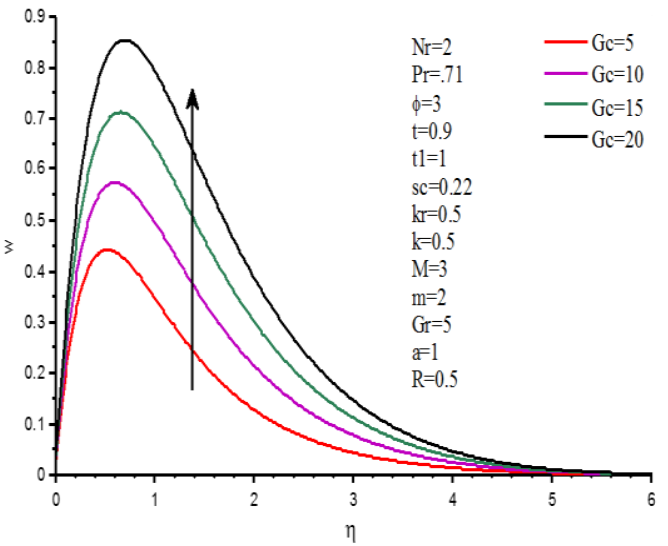

Fig. 3(b): Effect of Ge on w.

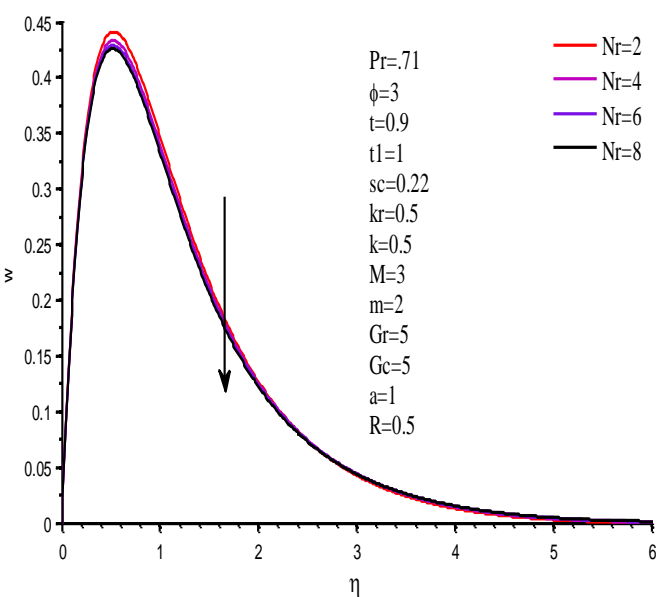

Fig. 4(b): Effect of $\mathrm{Nr}$ on w

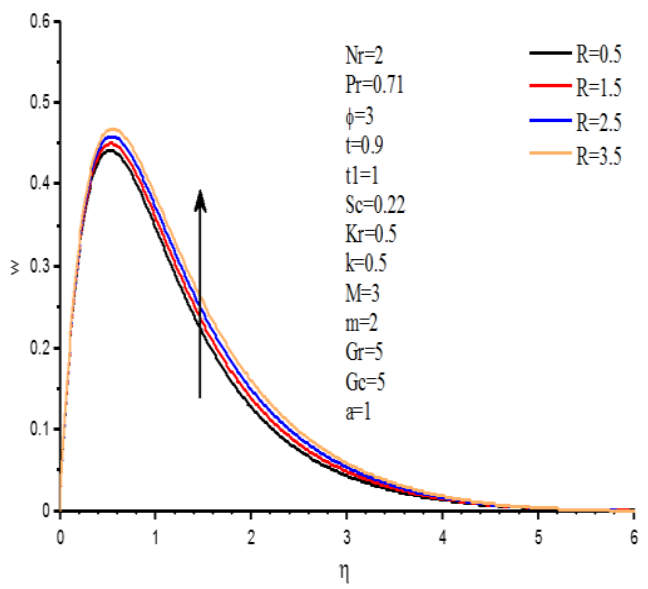

Fig. 5(b): Effect of R on w.

Fig. 6(a) and Fig. 6(b), depict the influence of heat absorption parameter on primary and secondary velocity of the fluid. The velocity of the fluid decreases with an increasing value of heat abs orption parameter $\phi$. Increase of heat absorption resists the flow and results in decreasing the velocity. It is perceived from Fig. 7(a) and Fig. 7(b) that $\mathrm{u}$ and $\mathrm{w}$ decrease on increasing values of Sc. Schmidt number is the ratio of kinematic viscosity to thermal 
diffusion which results in resisting the flow pattern and hence the velocity decreases. Fig. 8(a) and Fig. 8(b), demonstrate the effect of chemical reaction parameter on primary and secondary fluid velocities. It is evident from these two figures, that, $\mathrm{u}$ and $\mathrm{w}$ decrease on increasing $\mathrm{Kr}$. It is due to the natural fact that chemical reaction influence gives rise to decrement in velocity. Fig. 9(a) and Fig. 9(b) illustrate the effect of Hall current on the primary and secondary velocity of the fluid. It is evident that, $u$ and $w$ increase on increasing $\mathrm{m}$. This implies that Hall current tends to accelerate fluid flow in both the primary and secondary flow directions throughout the boundary layer region. This is due to the reason that, Hall current induces s econdary flow in the flow-field.

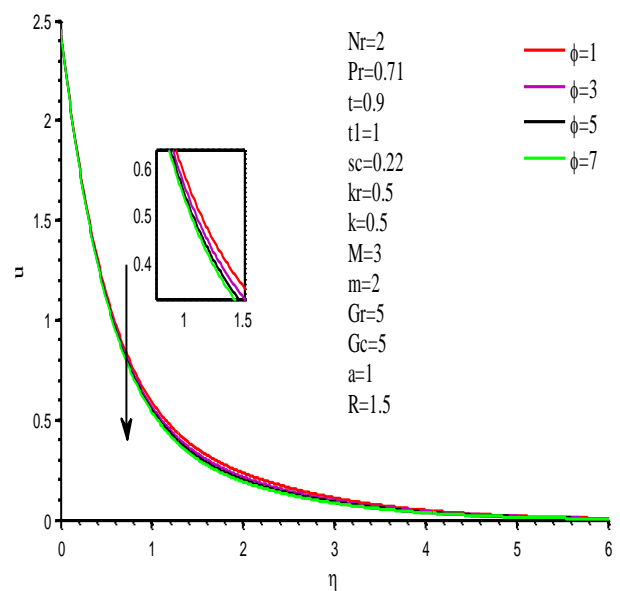

Fig. 6(a): Effect of $\phi$ on $u$.

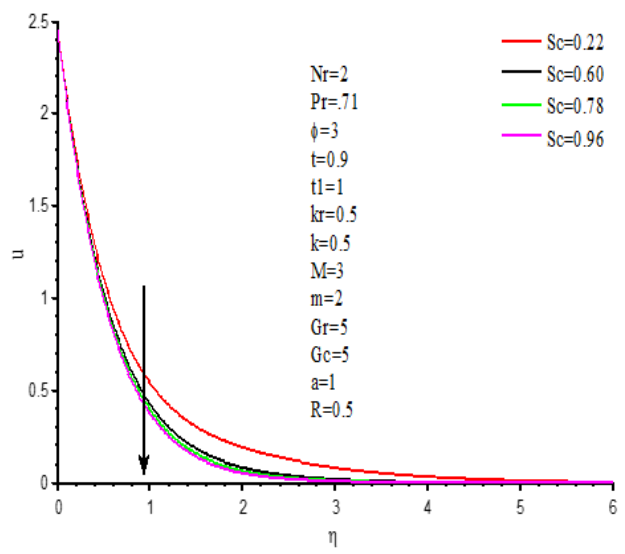

Fig. 7(a): Effect of Sc on u.

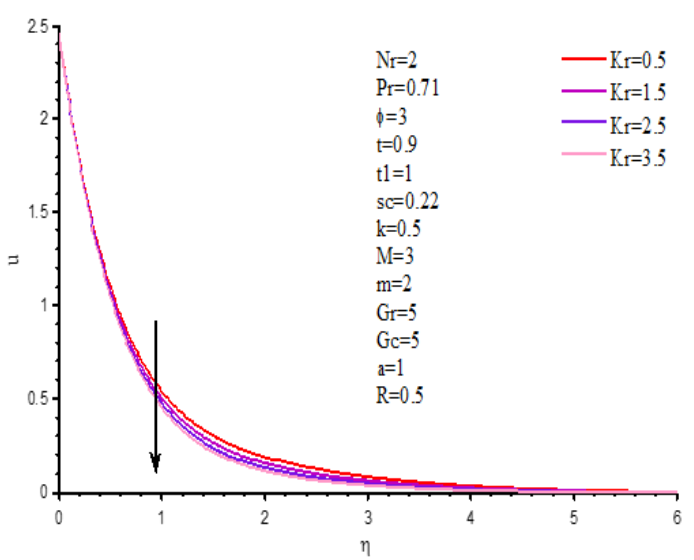

Fig. 8(a): Effect of Kr on u.

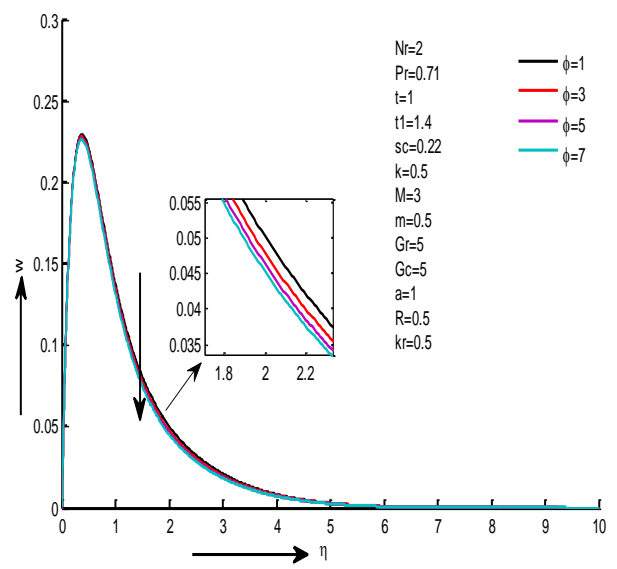

Fig. 6(b): Effect of $\phi$ on w.

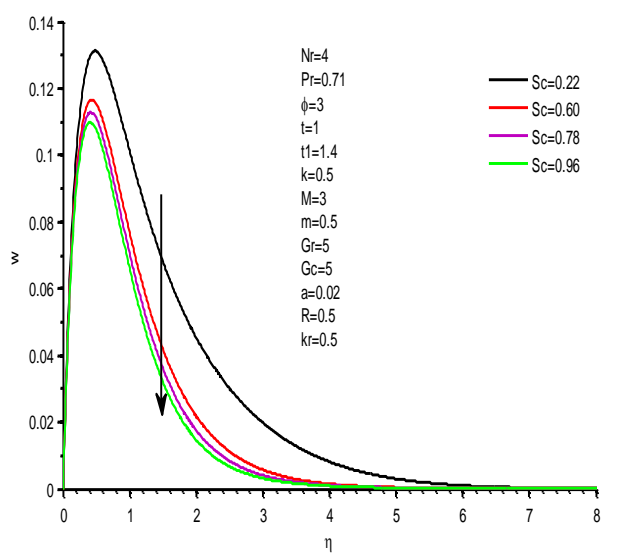

Fig. 7(b): Effect of Sc on w

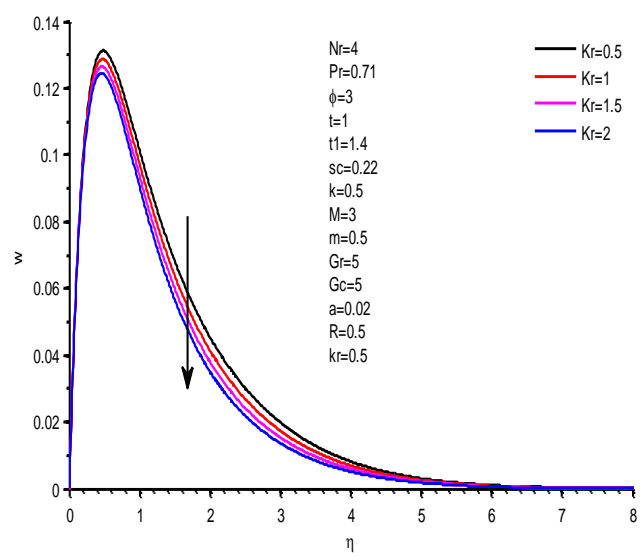

Fig. 8(b): Effect of Kr on w 


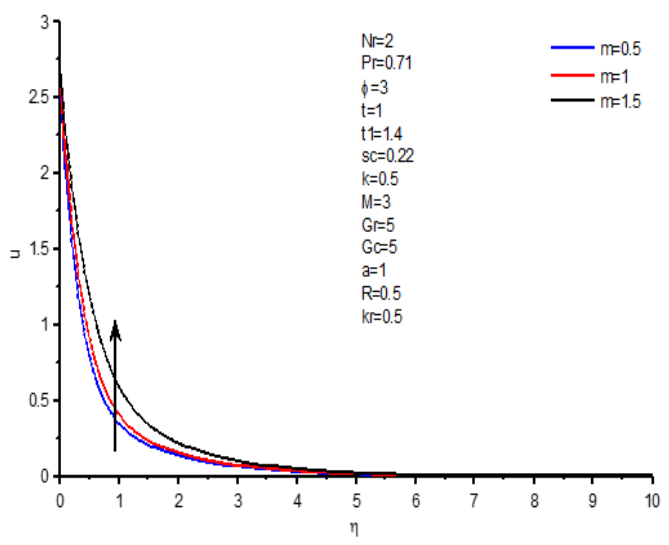

Fig. 9(a): Effect of $\mathrm{m}$ on $\mathrm{u}$.

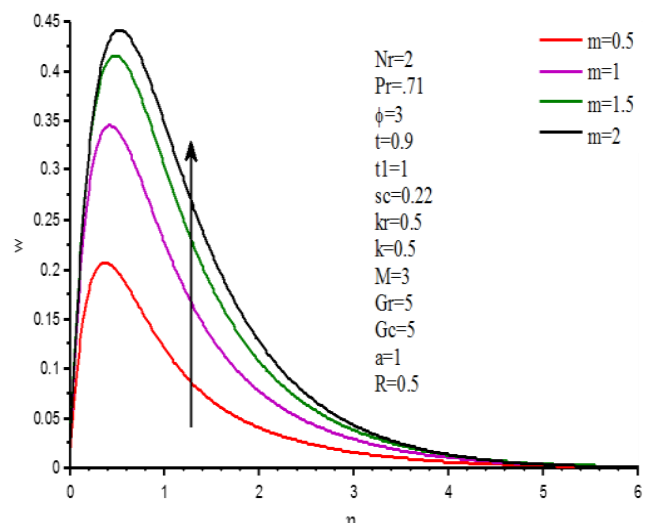

Fig. 9(b): Effect of $m$ on w.

Fig. 10(a) and Fig. 10(b) present the influence of porosity parameter on the primary and secondary velocity of the fluid. It is observed from these two figures that $\mathrm{u}$ and $\mathrm{w}$ increase with increase in $\mathrm{K}$. This is due to the known fact that fluid flow accelerates as the porousness enhances.

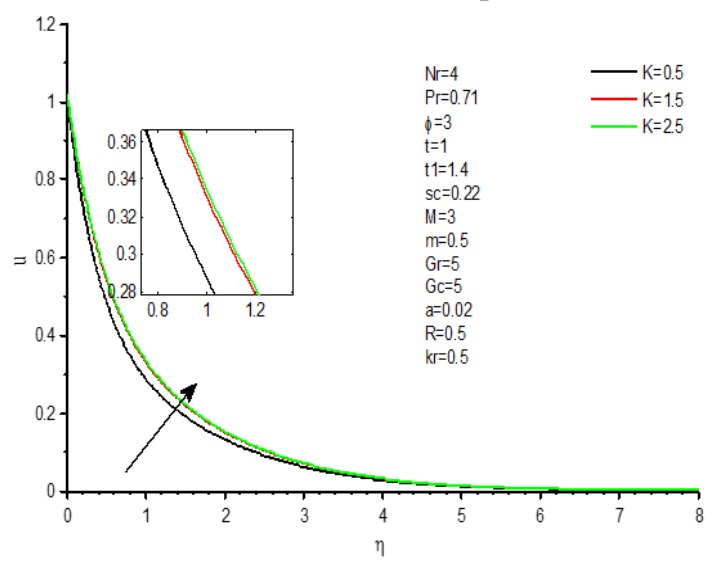

Fig. 10(a): Effect of K on u.

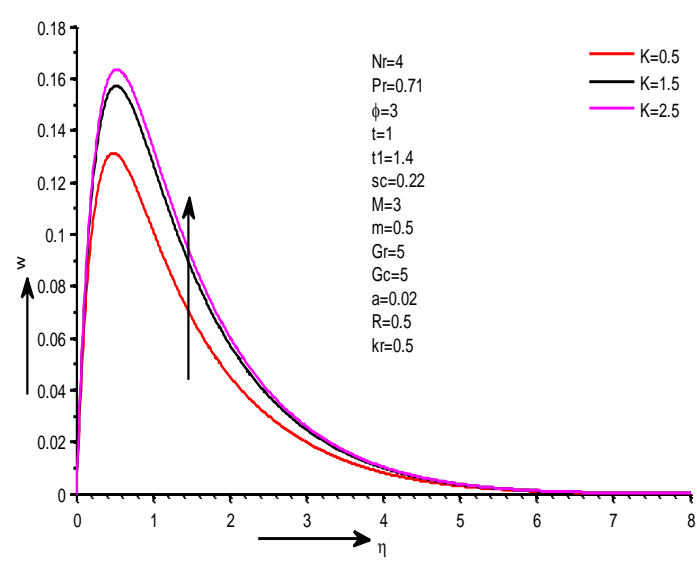

Fig. 10(b): Effect of K on w.

In order to examine the effect of critical time for rampedness and isothermal, numerical values of fluid temperature, $\mathrm{T}$ is computed from the analytical solution (19) and is shown graphically against boundary layer coordinate $\eta$ in Figs. 11-19. Fig. 11 illustrates the effect of radiation parameter on ramped temperature. It is evident from Fig. 11 that the temperature of the fluid increases for raising values of Nr. The reason behind this nature is due to the generation of heat due to radiation. Fig. 12 depicts the effect of heat absorption parameter on ramped temperature. It is noticed from these two figures that the temperature of the fluid decreases for increasing values of $\phi$.It is known fact that that heat absorption leads to reduce the heat of the fluid and hence the decrease in velocity. Fig. 13 exhibits the effect of Prandtl number on temperature. It is evident from the Fig. 13 that the temperature of the fluid decreases with increasing values of Prandtl number also represents the product of dynamic viscosity and specific heat capacity, divided by thermal conductivity of the primary fluid. Higher Prandtl fluid will therefore possess a much lower thermal conductivity and this will result in a significant decrease in temperatures in the boundary layer. It is perceived from the Fig. 14 that the temperature of the fluid increases for increasing values of radiation absorption R. From Fig. 15 it is noticed that the temperature of the fluid increases when time $\mathrm{t}$ increases. When $t>t_{1}$ the temperature become uniform and it is equal to 1 , which agrees with the condition (16). As we know that isothermal plate, temperature of the plate is uniform,i.e. in nondimensional form fluid temperature $T=1$ at the plate for every values of time $t$. This means that nature of fluid temperature is same for both ramped temperature and isothermal plate when $t>t_{1}$. However, fluid temperature is getting enhanced in the flow-field whether $t \leq t_{1}$ or $t>t_{1}$. It is evident in the Figs. 16-19. 


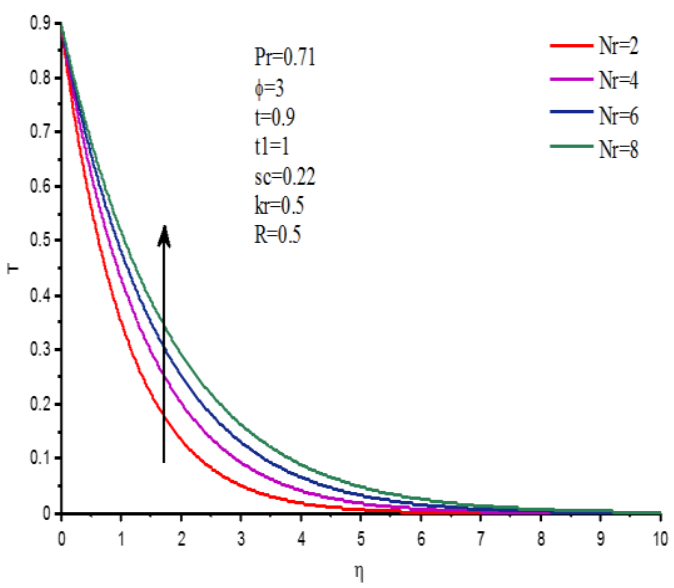

Fig.11: Effect of Nr on Temperature.

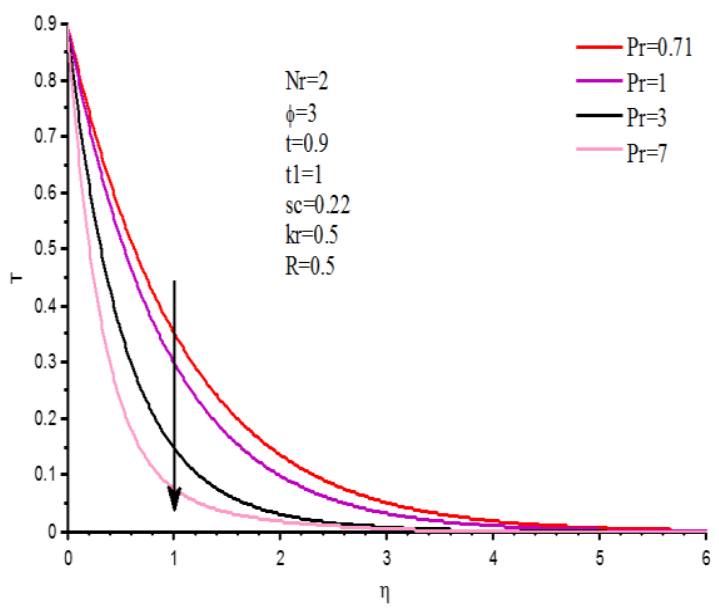

Fig.13: Effect of Pr on Temperature.

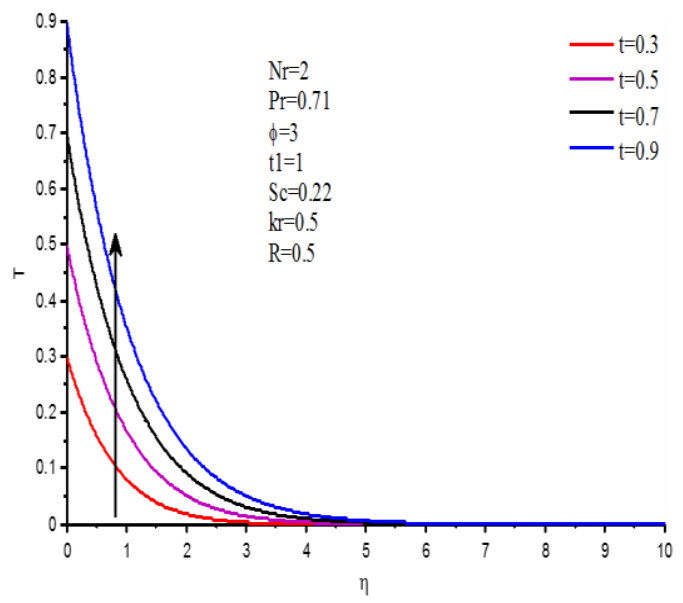

Fig.15: Effect of $\mathrm{t}$ on Temperature.

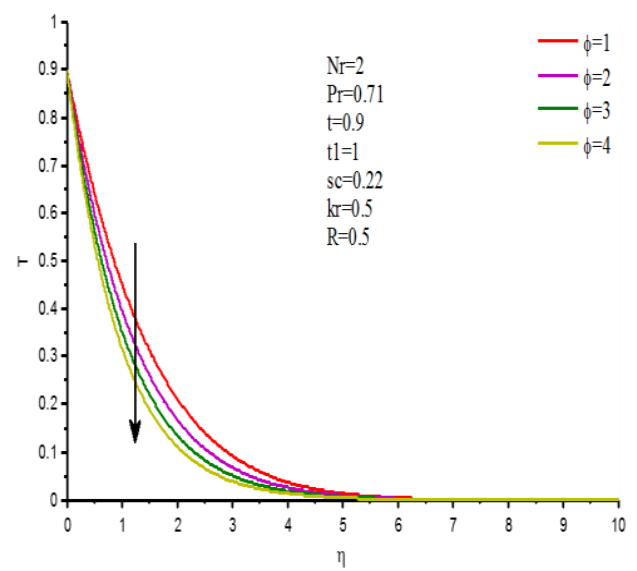

Fig.12: Effect of $\phi$ on Temperature.

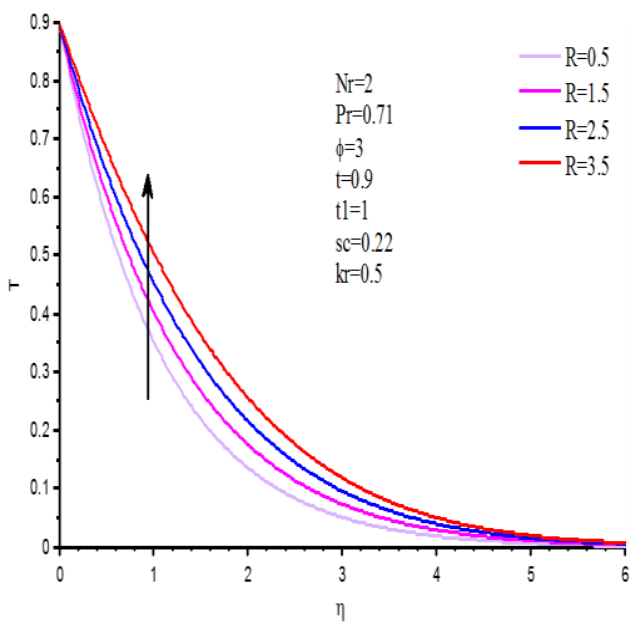

Fig.14: Effect of R on Temperature

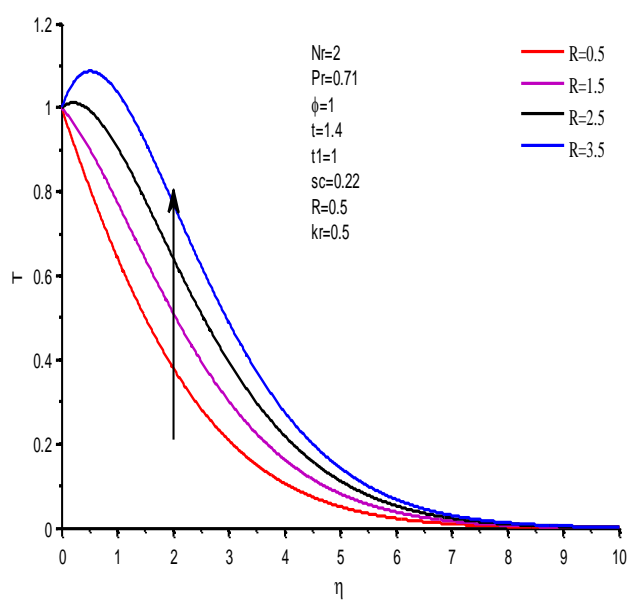

Fig.16: Effect of R on Temperature. 


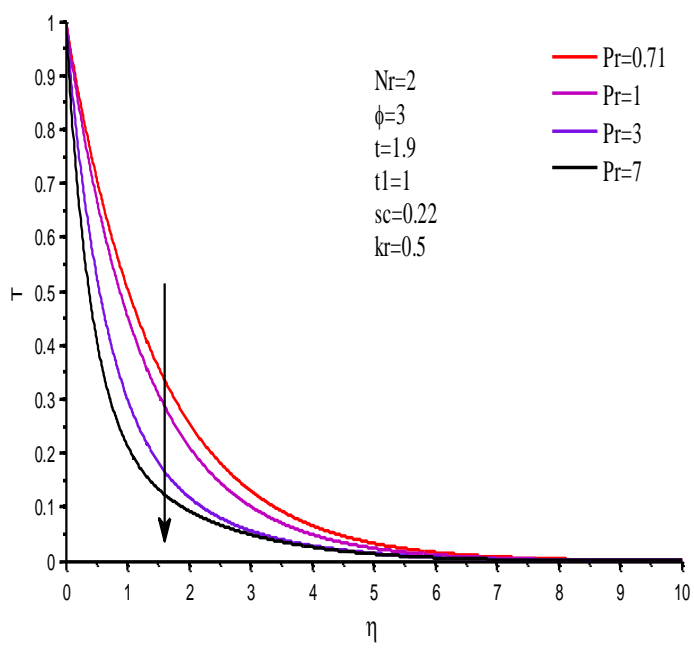

Fig. 17: Effect of Pr on Temperature.

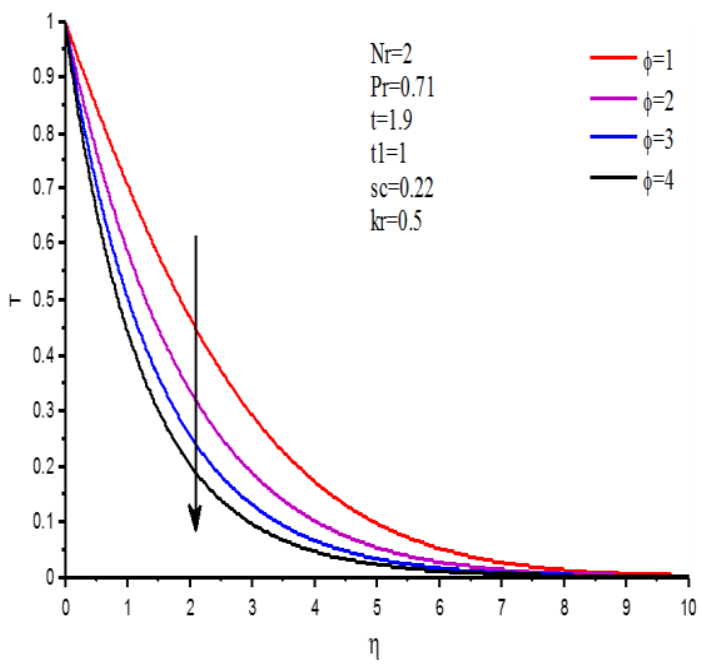

Fig. 19: Effect of $\phi$ on Temperature.

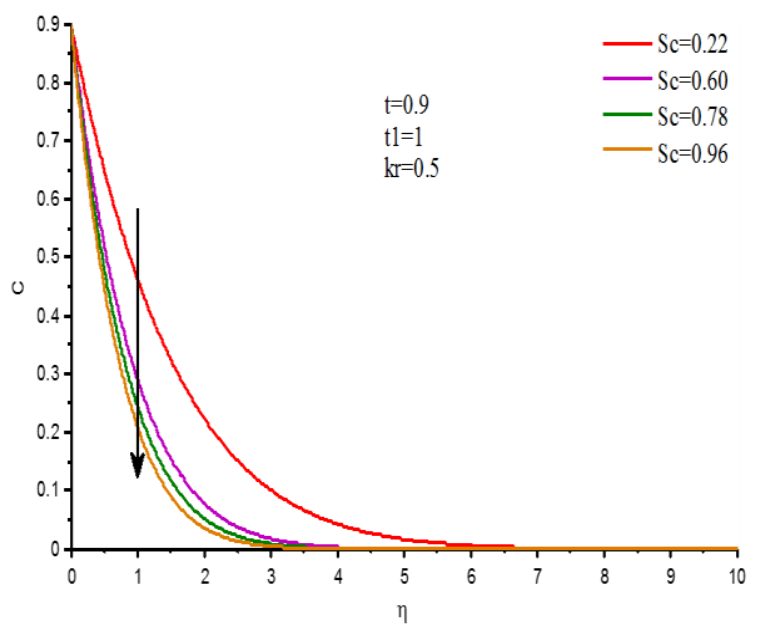

Fig. 21: Effect of Sc on Concentration

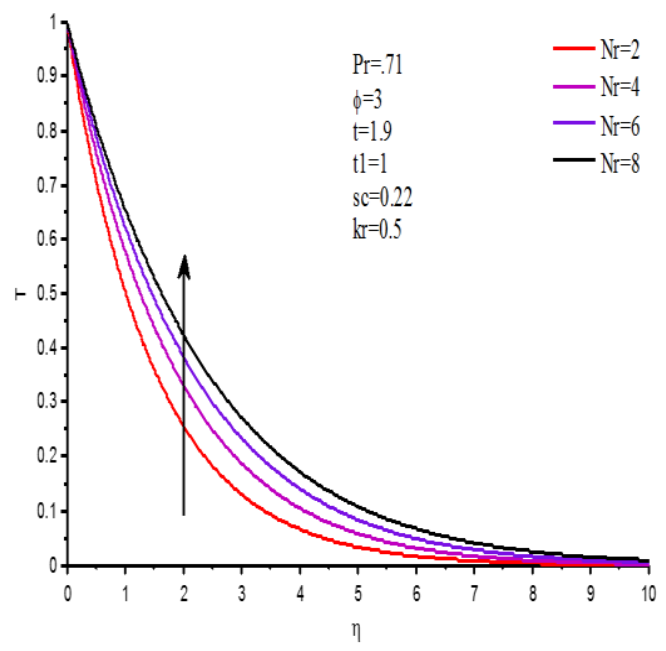

Fig. 18: Effect of $\mathrm{Nr}$

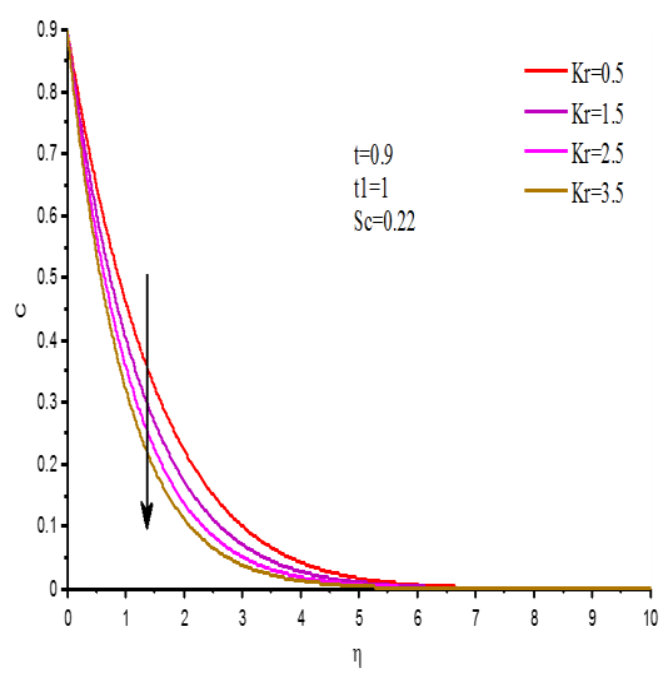

Fig. 20: Effect of $\mathrm{Kr}$ on Concentration.

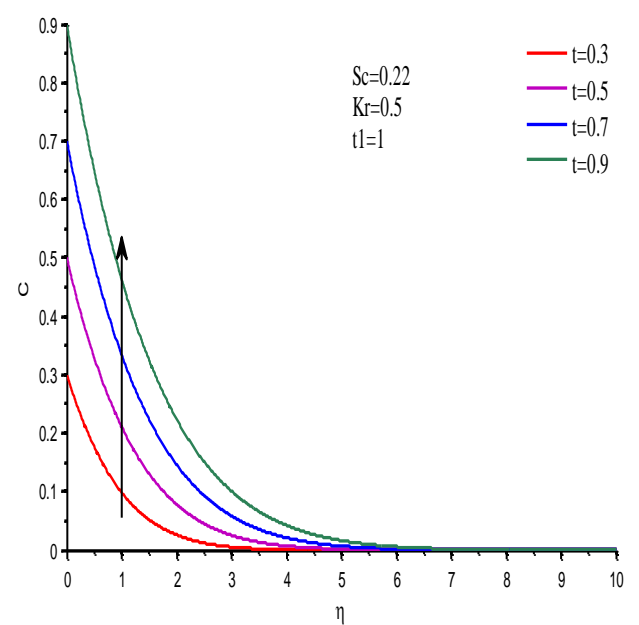

Fig. 22: Effect of $t$ on Concentration 
From Fig. 20 and Fig. 21, it is noticed that the concentration of the fluid decreases for increasing values of Kr and Sc. Sc represents the ratio of the mass (species) and viscous diffusion time sales. It is also the ratio of momentum diffusivity to species diffusivity. Higher values of Sc correspond to higher density species diffusing in air. Increasing Sc lowers the chemical molecular diffusivity of the species. As Sc is increased the concentration boundary layer will become relatively thinner than the viscous (momentum) boundary layer. Fig. 22 exhibits the variations in concentration of the fluid for different values of time $t$. It is evident from the figure that the concentration of the fluid increases for increasing values of time $t$.

The numerical values of rate of mass transfer at the plate i.e. $(\partial C / \partial \eta)_{\eta=0}$, computed from the analytical expression (21), are presented in the Table.1 for various values of Sc and Kr. Table. 1 exhibits that the rate of mass transfer at the plate increases for increasing values of $\mathrm{Sc}$ and $\mathrm{Kr}$.

Table 1: Variations in mass transfer, when $\mathrm{t}=0.9, \mathrm{t}_{1}=1$

\begin{tabular}{|c|c|c|}
\hline $\mathrm{Kr}$ & $\mathrm{Sc}$ & $\mathrm{Sh}$ \\
\hline 1 & 0.60 & 2.2931 \\
1.5 & 0.60 & 2.2959 \\
2.5 & 0.60 & 2.3433 \\
3.5 & 0.60 & 2.4221 \\
\hline 1.5 & 0.22 & 1.2193 \\
1.5 & 0.60 & 2.0136 \\
1.5 & 0.78 & 2.2959 \\
1.5 & 0.96 & 2.5470 \\
\hline
\end{tabular}

The numerical values of rate of heat transfer at the plate i.e. $(\partial T / \partial \eta)_{\eta=0}$, computed from the analytical expression (22), are presented in the Tables 2. From this Table it is noticed that $(\partial T / \partial \eta)_{\eta=0}$ increases on increasing $\operatorname{Pr}, \phi, \mathrm{Nr}$ whereas, it decreases on increasing $\mathrm{R}$.

Table 2: Variations in rate of heat transfer under the effect of several parameters, when $\mathrm{t}=0.9$

\begin{tabular}{|c|c|c|c|c|c|}
\hline $\mathrm{R}$ & $\mathrm{Nr}$ & $\Phi$ & $\mathrm{t}_{1}$ & $\mathrm{Pr}$ & $\mathrm{Nu}$ \\
\hline 0.5 & 4 & 3 & 1 & 7.1 & 1.6597 \\
1 & 4 & 3 & 1 & 7.1 & 1.4696 \\
1.5 & 4 & 3 & 1 & 7.1 & 1.2794 \\
\hline 0.1 & 4 & 3 & 1 & 0.71 & 0.1708 \\
0.1 & 6 & 3 & 1 & 0.71 & 0.4216 \\
0.1 & 8 & 3 & 1 & 0.71 & 0.3897 \\
\hline 0.1 & 6 & 1 & 1 & 0.71 & 0.6535 \\
0.1 & 6 & 3 & 1 & 0.71 & 0.4216 \\
0.1 & 6 & 5 & 1 & 0.71 & 0.5008 \\
\hline 0.1 & 4 & 3 & 1 & 0.71 & 0.6388 \\
0.1 & 4 & 3 & 1.5 & 0.71 & 0.4925 \\
0.1 & 4 & 3 & 2.0 & 0.71 & 0.4193 \\
\hline 0.5 & 4 & 3 & 1 & 0.71 & 1.0439 \\
0.5 & 4 & 3 & 1 & 3 & 1.3959 \\
0.5 & 4 & 3 & 1 & 5 & 1.6597 \\
\hline
\end{tabular}

The numerical values of shear stress $(\partial F / \partial \eta)_{\eta=0}$ at the plate computed from the analytical expression (23), are presented in the tabular form in Table. 3 for various values of $\mathrm{Nr}, \mathrm{M}, \mathrm{m}, \mathrm{R}, \mathrm{Gc}, \mathrm{K}, \phi, \mathrm{t}_{1}$. It is observed from Table. 3 that the shear stress at the plate decreases on increasing $\mathrm{t} 1, \mathrm{M}, \mathrm{m}, \mathrm{R}, \mathrm{K}$ and $\phi$ whereas it increases on increasing $\mathrm{Nr}$ and $\mathrm{Gc}$. 
Table 3: Variations in skin friction under the effect of several parameters, when $\mathrm{t}=0.9, \mathrm{Sc}=0.22, \mathrm{a}=1, \mathrm{Kr}=0.5$

\begin{tabular}{|c|c|c|c|c|c|c|c|c|}
\hline $\mathrm{M}$ & $\mathrm{Gr}$ & $\mathrm{Gc}$ & $\mathrm{M}$ & $\mathrm{R}$ & $\mathrm{K}$ & $\mathrm{Nr}$ & $\phi$ & $\tau$ \\
\hline 3 & 5 & 5 & 0.5 & 0.5 & 0.5 & 4 & 3 & 7.5454 \\
3.5 & 5 & 5 & 0.5 & 0.5 & 0.5 & 4 & 3 & 8.5927 \\
4 & 5 & 5 & 0.5 & 0.5 & 0.5 & 4 & 3 & 9.6681 \\
\hline 3 & 5 & 5 & 0.5 & 0.5 & 0.5 & 4 & 3 & 7.5454 \\
3 & 10 & 5 & 0.5 & 0.5 & 0.5 & 4 & 3 & 7.8513 \\
3 & 15 & 5 & 0.5 & 0.5 & 0.5 & 4 & 3 & 8.1573 \\
\hline 3 & 5 & 5 & 1.5 & 0.1 & 0.5 & 4 & 3 & 8.1487 \\
3 & 5 & 10 & 1.5 & 0.1 & 0.5 & 4 & 3 & 8.4546 \\
3 & 5 & 15 & 1.5 & 0.1 & 0.5 & 4 & 3 & 8.7606 \\
\hline 3 & 5 & 5 & 0.5 & 0.5 & 0.5 & 4 & 3 & 7.5454 \\
3 & 5 & 5 & 1 & 0.5 & 0.5 & 4 & 3 & 6.5027 \\
3 & 5 & 5 & 1.5 & 0.5 & 0.5 & 4 & 3 & 5.6323 \\
\hline 3 & 5 & 5 & 0.5 & 0.5 & 0.5 & 4 & 3 & 7.5454 \\
3 & 5 & 5 & 0.5 & 1 & 0.5 & 4 & 3 & 6.7565 \\
3 & 5 & 5 & 0.5 & 1.5 & 0.5 & 4 & 3 & 5.9677 \\
\hline 3 & 5 & 5 & 0.5 & 1 & 0.5 & 4 & 3 & 6.7565 \\
3 & 5 & 5 & 0.5 & 1 & 1 & 4 & 3 & 6.2958 \\
3 & 5 & 5 & 0.5 & 1 & 1.5 & 4 & 3 & 6.1361 \\
\hline 3 & 5 & 5 & 0.5 & 0.5 & 0.5 & 2 & 3 & 7.5454 \\
3 & 5 & 5 & 0.5 & 0.5 & 0.5 & 4 & 3 & 8.1487 \\
3 & 5 & 5 & 0.5 & 0.5 & 0.5 & 6 & 3 & 8.1922 \\
\hline 3 & 5 & 5 & 0.5 & 0.5 & 0.5 & 4 & 1 & 7.8053 \\
3 & 5 & 5 & 0.5 & 0.5 & 0.5 & 4 & 3 & 7.5454 \\
3 & 5 & 5 & 0.5 & 0.5 & 0.5 & 4 & 5 & 6.1353 \\
\hline
\end{tabular}

\section{Conclusions}

An investigation of analytical study of ramped temperature influence on MHD convective chemical reactive and heat absorbing fluid past an exponentially accelerated vertical plate is carried out. Important findings of the problems are cited below.

1. Primary and secondary velocities of the fluid flows are increasing for Gr, Gc, R, m, K whereas in the case of $\mathrm{Nr}, \phi, \mathrm{Sc}, \mathrm{Kr}$ the primary and secondary velocities are decreasing.

2. Ramped temperature of the fluid is falling down for increasing values of $\operatorname{Pr}, \phi$ and the temperature rising for increasing values for $\mathrm{Nr}, \mathrm{R}, \mathrm{t}$.

3. Concentration of the fluid decreases for increasing values of $\mathrm{Sc}$ and $\mathrm{Kr}$ but it shows reverse effect for $\mathrm{t}$.

4. Coefficient of skin friction increases due to the increments in $\mathrm{Gc}, \mathrm{Nr}$ and decreases for the increments in $\mathrm{M}, \mathrm{m}, \mathrm{R}, \mathrm{K}, \phi$.

5. Coefficient of rate of heat transfer increases for the increasing values of Pr, $\phi$ whereas it gives opposite reaction for the parameters $\mathrm{R}, \mathrm{Nr}$.

6. Coefficient of rate of the mass transfer increases for increasing values of Sc and $\mathrm{Kr}$.

\section{References}

Barik, R. N. (2014): Chemical reaction and radiation effects of MHD free convective flow past an impulsively moving vertical plate with ramped wall temperature and concentration, European Journal of Advances in Engineering and Technology, 1, 56-68.

Devi, A. S. P., Kandasamy, R. (2010): Effects of a chemical reaction heat and mass transfer on MHD flow past a semi-infinite plate, Z. Angew. Math. Mech, 80, 697-701. https://doi.org/10.1002/1521-4001 
Hossain, M. A., Vafai, K., Khalil. K. (2001): The effect of radiation on free convection flow of fluid with variable viscosity from a porous vertical plate, Int. J. Therm. Sci, 40, 115-124. https://doi.org/10.1016/S1290$\underline{0729(00) 01200-X}$

Kundu, P. K., Das, K., Acharya, N. (2014): Flow features of a conducting fluid near an accelerated vertical plate in porous medium with ramped wall temperature, J. Mech. 30, 277-288. https://doi.org/10.1017/jmech.2014.14 Mabood, F., Shateyi, S., Rashidi, M. M., Momoniat, E., Freidoonimehr, N. (2016): MHD stagnation point flow heat and mass transfer of nano fluids in porous medium with radiation, viscous dissipation and chemical reaction, Advanced Powder Technology 27 742-749. https://doi.org/10.1016/j.apt.2016.02.033

Manglesh, A., and Gorla, M. G. (2013): MHD free convective flow through porous medium in the presence of Hall current, radiation and thermal diffusion, Indian J. Pure Appl.Maths, 44 ,743-756.

https://link.s pringer.com/article/10.1007/s 13226-013-0040-9

Narahari, M. (2012): Transient free convection flow between long vertical parallel plates with ramped wall temperature at one boundary in the presence of thermal radiation and constant mass diffusion, Meccanica, 47, 1961-76. https://link.s pringer.com/article/10.1007/s 11012-012-9567-9

Narayan, P. V. S., Rami Reddy, G., Venkataramana, S. (2011): Hall current effects on free-convection MHD flow fast a porous plate, Int J Automot Mech Eng, 3, 350-63.https://doi.org/10.15282/ijame.3.2011.10.0029.

Narayan, P. V. S., Venkateswarlu, B., Venkataramana, S. (2014): Effects of Hall current and radiation absorption on MHD micro polar fluid in a rotating system, Ain Shams Engineering Journal, 4, 843-854. https://doi.org/10.1016/j.as ej.2013.02.002

Pop, I., Watanabe, T. (1994): Hall effects on magneto hydrodynamic free convection about a semi-infinite vertical flat plate, Int. J. Eng. Sci. 32, 1903-1911.https://doi.org/10.1016/0020-7225(94)90087-6

Prasad, V. R., and Reddy, N.B. (2008): Radiation effects on an unsteady MHD convective heat and mass transfer flow past a semi-infinite vertical permeable moving plate embedded in a porous medium, Journal of Energy Heat and Mass Transfer, 30, 57-68.

Raju, M. C., Varma, S. V. K. (2014): Soret effects due to natural convection in a non-Newtonian fluid flow in porous medium with heat and mass transfer, Journal of Naval Architecture and Marine Engineering, 11 47-156 https:/doi.org/10.3329/jname.v11i2.17563.

Raju, R. S., Reddy, G. J., Rao, J. A. Rashidi, M. M., Gorla, R. S. R. (2016): Analytical and numerical study of unsteady MHD free convection flow over an exponentially moving vertical plate with heat absorption, International Journal of Thermal Sciences, 107, 303-315.https://doi.org/10.1016/j.ijthermalsci.2016.04.005

Reddy, G. V. R., Bhaskar Reddy, N., and Gorla, R. S. R. (2016): Radiation and chemical reaction effects on MHD flow along a moving vertical porous plate, Int. J. of Applied Mechanics and Engineering, 21, 157-168. https://doi.org/10.1515/ijame-2016-0010

Rout, P. K., Sahoo, S. N., and Dash, G. C. (2016): Effect of heat source and chemical reaction on MHD flow past a vertical plate with variable temperature, Journal of Naval Architecture and Marine Engineering, 13, 10111. https://doi.org/10.3329/jname.v13i1.23930

Saha, L. K., Siddiqa S.,Hossain, M. A. (2011): Effect of Hall current on MHD natural convection flow from vertical permeable flat plate with uniform surface heat flux, Appl Math Mech Engl. 32, $1127-46$. https://link.springer.com/article/10.1007/s 10483-011-1487-9

Seth, G. S., Kumbhakar, B., Sharma1, R. (2016): Unsteady MHD free convection flow with Hall effect of a radiating and heat absorbing fluid past a moving vertical plate with variable ramped temperature, Journal of the Egyptian Mathematical Society, 24 ,471-478. https://doi.org/10.1016/j.joems.2015.07.007

Seth, G. S., Sarkar, S., and Sharma, R. (2016): Effects of Hall current on unsteady hydro magnetic free convection flow past an impulsively moving vertical plate with Newtonian heating, Int. J. of Applied Mechanics and Engineering, 21, 187-203. https://doi.org/10.1515/ijame-2016-0012

Seth, G. S., Sarkar, S., Hussain, S. M. (2014): Effects of Hall current, radiation and rotation on natural convection heat and mass transfer flow past a moving vertical plate, Aim Shams Engineering Journal, 5, 489503 . https://doi.org/10.1016/j.asej.2013.09.014

Seth, G. S., Ansari, M. S., and Nandkeolyar, R. (2011): MHD natural convection flow with radiative heat transfer past an impulsively moving plate with ramped wall temperature, Heat Mass Transfer, 47, 551-561, https://link.springer.com/article/10.1007/s00231-010-0740-1

Singh, R. K., and Singh, A. K. (2010): Transient MHD free convective flow near a semi- infinite vertical wall having ramped temperature, Int. J. of Appl. Math and Mech. 6, 69-79.

Srinivas, S., Reddy, P. B. A., and Prasad, B. S. R. V. (2014): Effects of chemical reaction and thermal radiation on MHD flow over an inclined permeable stretching surface with non-uniform heat source/sink: an application 
to the dynamics of blood flow, Journal of Mechanics in Medicine and Biology, 14. https://doi.org/10.1142/S0219519414500675

Srinivasacharya, D., and Reddy, G. S. (2016): Chemical reaction and radiation effects on mixed convection heat and mass transfer over a vertical plate in power-law fluid saturated porous medium, Journal of the Egyptian Mathematical Society, 24, 108-115. https://doi.org/10.1016/j.joems.2014.10.001

Srinivasacharya, D., and Swamy Reddy, G. (2013): Free convection in a non-Newtonian power law Fluid saturated porous medium with Chemical reaction and radiation effects, Special Topics \& Reviews in Porous Media - An International Journal, 4, 223-236. DOI: 10.1615/SpecialTopics RevPorous Media.v4.i3.30

Tripath, R. S., Dash, G. C., Mishra, S. R., Baag, S. (2015): Chemical reaction effect on MHD free convective surface over a moving vertical plate through porous medium, Alexandria Engineering Journal, 54, 673-679. https://doi.org/10.1016/j.aej.2015.04.012 